\title{
Transfert hydrique dans un sol argileux gonflant non saturé : influence du confinement
}

S. ROLLAND

D. STEMMELEN

C. MOYNE

LEMTA, UMR 7563

CNRS-INPL-UHP

2, av. de la Forêt-

de-Haye, BP 160

54500 Vandoeuvre-

lès-Nancy

Sebastien.Rolland@ensem. inpl-nancy.fr

Didier.Stemmelen@ensem. inpl-nancy.fr

Christian.Moyne@ensem. inpl-nancy.fr

F. MASROURI

LAEGO-ENSG-INPL rue du Doyen-Roubault BP 40

54501 Vandœuvrelès-Nancy

Farimah.Masrouri@ensg. impl-nancy.fr

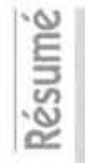

La description des mécanismes d'infiltration et de gonflement dans les sols argileux représente un enjeu important en géotechnique, en agronomie ou dans l'industrie pétrolière. L'objectif de cet article est d'analyser les effets de couplage hydromécanique lors de l'imbibition d'un milieu argileux gonflant soumis à différentes conditions de confinement. Le matériau utilisé est un mélange de bentonite et de limon de Xeuilley. Préparé à une teneur en eau massique connue, ce mélange est compacté au moyen d'une technique doublepiston, permettant d'élaborer des éprouvettes de sol d'humidité et de masse volumique sèche uniformes. Des essais d'imbibition capillaire ont été réalisés pour trois types de confinement : libre, œdométrique, à volume constant. La technique de gammamétrie double-source est utilisée afin de suivre localement, et de manière non intrusive, l'évolution simultanée de la masse volumique sèche et de la teneur en eau. Une comparaison des trois cinétiques d'humidification est finalement menée à partir d'une caractérisation en terme de diffusivité hydrique, basée sur une description lagrangienne des transferts.

Mots-clés: sol argileux, milieux poreux déformables, compactage, imbibition, gonflement, humidité, gammamétrie double-source, diffusivité, confinement.
NDLR: Les discussions sur cet article sont acceptées jusqu'au $1^{\text {er }}$ février 2004
The description of infiltration and swelling mechanisms in clayey soils is of significant interest in geotechnics, agronomy or petroleum industry. The aim of this article is to show the effects of hydramechanical couplings during imbibition in a compacted sweiling clay sample, under different confinement conditions. The material is a bentonite-silt mixture, prepared with a known water content and compacted with a dual piston technique. This method allows to produce homogeneous soil samples, in terms of water content and dry bulk density. Experiments related to bottom infiltration are then carried out with three different mechanical boundary conditions: free, oedometric, fixed volume. The non-intrusive dual-energy gamma-ray technique is used to measure the local variation of bulk density and humidity. Finally, the three infiltration kinetics are compared in terms of hydraulic diffusivity, described in a Lagrangian way.

Key words : clayey soil, deformable porous media, compaction, imbibition, swelling, water content, dual-energy gamma-ray technique, diffusivity, confinement. 


\section{Notations}

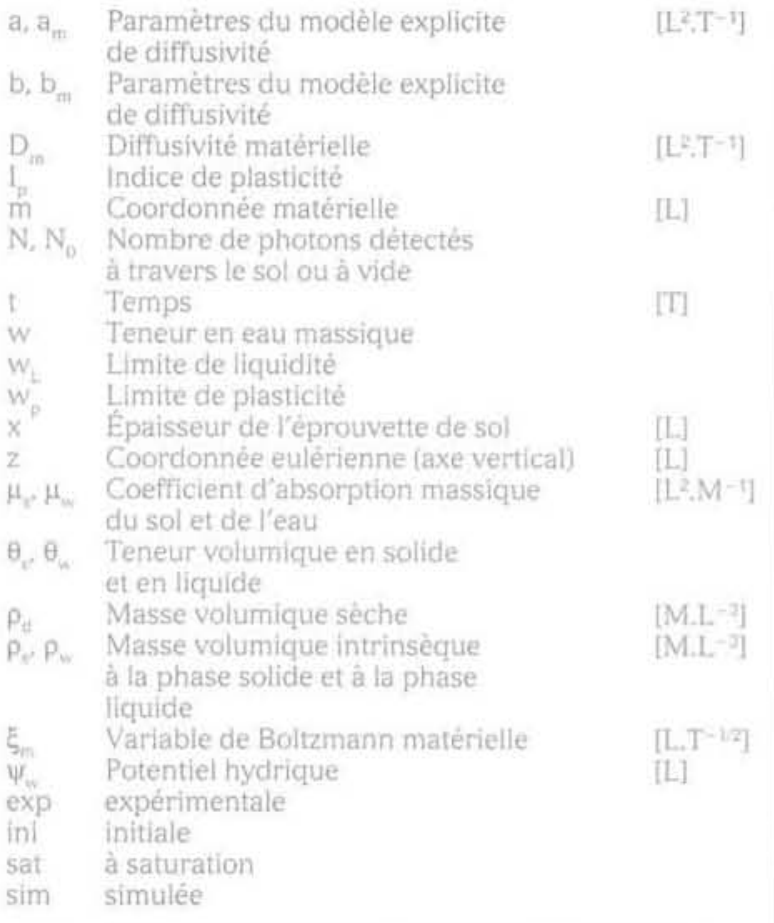

\section{1}

\section{Introduction}

De par leur abondante présence dans les sols, la grande diversité de leur nature et de leurs propriétés intrinsèques, les argiles font l'objet de nombreux travaux de recherche appliquée. Dans le domaine de la géotechnique, en particulier, elles sont connues pour leur déformabilité, car elles sont susceptibles de gonfler ou de se rétracter suivant leur degré d'humidité. Il est alors nécessaire d'en tenir compte pour les constructions, afin d'éviter l'apparition d'effets néfastes (Philipponnat, 1991; Vandangeon, 1992; Derriche et al., 1999). De plus, les sols argileux se caractérisent par une faible perméabilité. Cela explique qu'ils soient souvent utilisés en tant que constituants pour la réalisation de barrières étanches dans des sites de stockage. Il apparaît donc essentiel de bien connaitre les propriétés hydrauliques et mécaniques de ces milieux, afin de savoir maitriser leur comportement.

L'objectif du travail présenté ici est de montrer, au moins du point de vue qualitatif, les effets du couplage hydromécanique lors de l'imbibition d'eau dans des milieux argileux gonflants soumis à trois conditions de confinement différentes: libre, œdométrique et à volume constant.

Nous vous proposons tout d'abord de faire une présentation succincte du matériau utilisé, à travers ses constituants et ses caractéristiques géotechniques, ainsi que de la technique de réalisation des échantillons. Notre cellule d'essai permettant de réaliser des imbibitions pour différents degrés de confinement est ensuite décrite avec l'ensemble de son environnement.

Dans l'argile, les transferts hydriques s'accompagnent localement de modification de la masse volu- mique sèche: à tout instant deux grandeurs doivent donc être déterminées, ce qui nécessite l'utilisation d'un dispositif expérimental approprié. Actuellement, plusieurs techniques non intrusives permettent de répondre au problème. On trouve entre autres le synchrotron X (Garnier et al., 1998), la résonance magnétique nucléaire (Augier et al., 2002), techniques très performantes mais aussi très coûteuses; la gammamétrie double-source, méthode un peu plus ancienne, mais plus souple d'utilisation, sera utilisée pour notre étude.

Les résultats issus de ces techniques permettent ensuite d'accéder aux propriétés hydrauliques du matériau. Deux modes de description sont généralement adoptés en mécanique des milieux continus: la description eulérienne (Nakano et al., 1986; Angulo et al., 1993) et la description lagrangienne (Philip, 1968; Smiles et Rosenthal, 1968; Angulo et al., 1993). Du point de vue pratique, la première présente des avantages évidents puisque les mesures réalisées le sont dans un repère fixe. Elle est cependant physiquement critiquable, notamment parce qu'elle fait intervenir des termes diffusifs liés à la phase solide (Kirby et Smiles, 1999), Notre choix s'est donc orienté préférentiellement vers l'approche lagrangienne.

Après avoir brièvement validé notre méthodologie sur un milieu poreux de référence (béton cellulaire), nous présentons les résultats obtenus sur des échantillons de sol argileux gonflant. Une comparaison des trois cinétiques d'infiltration est finalement menée à partir d'une caractérisation en terme de diffusivité hydraulique, basée sur une description lagrangienne des transferts.

\section{2}

\section{Matériau et mise en forme}

\section{1}

\section{Caractéristiques du matériau}

Le sol déformable que nous avons ètudié est un matériau reconstitué en laboratoire à partir de deux sols fins. De la bentonite et du limon de Xeuilley sont mélangés, humidifiés et compactès à une pression de compactage donnée.

\section{Lasia \\ La bentonite}

La bentonite utilisée est une argile industrielle très plastique d'appellation commerciale "bentonite FVO n. Une analyse par diffraction aux rayons X montre qu'elle est composée de smectite dioctaédrique de type montmorillonite à compensation calcique, et qu'elle contient 5 à $10 \%$ de quartz et $1 \%$ de calcite. Une analyse par spectroscopie infrarouge confirme cette détermination et met aussi en évidence des traces de kaolinite. Nous avons mesuré une masse volumique des grains valant $\rho_{\mathrm{s}}=2,66 \pm 0,01 \mathrm{~g} \cdot \mathrm{cm}^{-3}$ par pycnométrie à l'hélium.

\section{(1) sithe \\ Le limon de Xeuilley}

Le limon utilisé dans ce travail est un limon argileux très plastique prélevé à Xeuilley (situé à $20 \mathrm{~km}$ au sud 
de Nancy). L'analyse par diffraction X montre que ce limon contient environ $60 \%$ de quartz, $20 \%$ de montmorillonite, $11 \%$ de feldspath, quelques $\%$ de kaolinite et de mica. Nous avons mesuré pour ce matériau une masse volumique des grains égale à $\rho_{\mathrm{s}}=2,69 \pm$ $0,01 \mathrm{~g} \cdot \mathrm{cm}^{-3}$ par pycnométrie à l'hélium.

Nous avons réuni dans le tableau I les valeurs moyennes des limites d'Atterberg mesurées pour ces deux constituants.

TABLEAUI Caractéristiques des matériaux utilisés: limites d'Atterberg.

Characteristics of the used materials Atterberg's limits.

\begin{tabular}{l|c|c|c} 
& $w_{f}(\%)$ & $w_{p}(\%)$ & $I(\%)$ \\
\hline Limon de Xeuilley & 53 & 30 & 23 \\
\hline Bentonite FVO & 164 & 64 & 100 \\
\hline
\end{tabular}

\section{Thing}

\section{Protocole du mélange et du compactage}

Nous avons choisi de mélanger manuellement $60 \%$ de bentonite et $40 \%$ de limon sous forme de poudres sèches, puis d'ajouter très progressivement la quantité d'eau nécessaire tout en malaxant le matériau pour obtenir une teneur en eau massique, w, de $25 \%$. Le mélange a ensuite été compacté, en appliquant une pression de compactage de $1 \mathrm{MPa}$, par une technique double-piston (Mesbah et al., 1999). Plusieurs essais ont été réalisés en élaborant le matériau par compactage statique, simple piston, de couches d'épaisseur 1 à $2 \mathrm{~cm}$. Compte tenu de l'hétérogénéité finale des échantillons obtenus par cette méthode, nous avons pris le parti de ne présenter dans cet article que les résultats relatifs aux échantillons compactés par la méthode double-piston Ainsi ont pu être élaborées des éprouvettes de sol relativement uniformes, de diamètre $11,2 \mathrm{~cm}$, de hauteur $12,0 \mathrm{~cm}$ et de masse volumique sèche $\rho_{\mathrm{d}}$ égale à $1,50 \mathrm{~g} \cdot \mathrm{cm}^{-3}$. Nous noterons que la combinaison $\rho_{\mathrm{d}}=1,50 \mathrm{~g} \cdot \mathrm{cm}^{-3}$ et $\mathrm{w}=25 \%$, facilement reproductible, correspond sensiblement au maximum de la courbe de compactage du mélange rẻalisée par Tabani (1999) (Fig. 1). Cette proportion permet sous charge

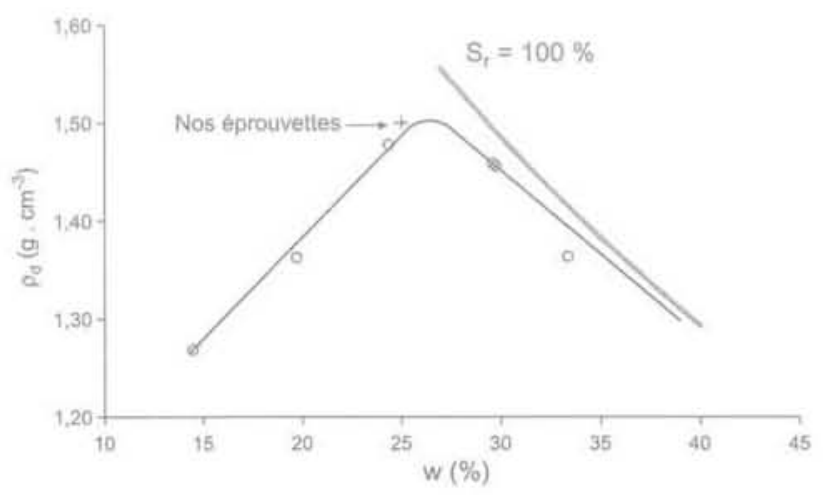

FIG.1 Courbe de compactage du mélange bentonite-limon (d'après Tabani, 1999).

Compaction curve of the bentonite-silt mixture (from Tabani, 1999). nulle d'obtenir un gonflement axial d'environ $25 \%$ ou d'obtenir une pression de gonflement de $630 \mathrm{kPa}$. C'est a priori un milieu bien adapté à la mesure par gammamétrie, pour laquelle les phénomènes doivent varier raisonnablement en amplitude ou en temps. De plus, la conductivité hydraulique du matériau n'est pas excessivement faible (voisine de $10^{-11} \mathrm{~m} / \mathrm{s}$ ), ce qui permet de réaliser différentes expériences dans des délais raisonnables (moins de trois mois)

Un carottage est ensuite réalisé pour obtenir des échantillons de diamètre plus adapté à la technique gammamétrique. Cette dernière étape gênère une quantité importante de matériau inutilisable pour la suite des essais. Cette quantité est en partie récupérée pour contrôler l'état initial de l'échantillon: mesure par pesée de la valeur de la teneur en eau massique $\left\langle w^{\text {inl }}\right\rangle$ (méthode par étuvage) et de la masse volumique sèche $\left\langle p_{d}^{i n i}\right\rangle$ (méthode par immersion dans le kerdane).

\section{0}

\section{Trois types de confinement}

Au cours de ce travail, nous avons réalisé un certain nombre d'essais d'imbibition sur le mélange gonflant, en faisant varier les conditions aux limites mécaniques. Pour ce faire, nous avons dû concevoir une cellule expérimentale instrumentée. Elle est formée d'une base circulaire en inox jouant le rôle de réservoir, facilement alimentèe en eau. Afin d'assurer des conditions de type œdométrique, l'éprouvette de sol étudiée est maintenue dans un tube en Plexiglas de diamètre interne $6 \mathrm{~cm}$, lui-même chemisé par un tube en inox. Ces deux tubes s'encastrent sans difficulté dans la base précédemment décrite. L'étanchéité entre la base et les tubes est assurée par deux joints toriques graissés. Une pierre poreuse de diamètre $6 \mathrm{~cm}$ et d'épaisseur $1 \mathrm{~cm}$ permet de réaliser le contact entre le sol et l'eau du réservoir. Il convient de préciser ici que tous les essais ont été réalisés sous charge d'eau nulle.

Pour le premier essai, nommé essai libre, l'éprouvette de sol est libre de se déformer axialement et latéralement. Un simple film plastique enveloppe l'éprouvette afin de limiter le phénomène d'évaporation. Pour cet essai, un capteur de déplacement et un micromètre à balayage laser permettent de mesurer respectivement les gonflements axial et radial de l'éprouvette.

Pour le deuxième essai, ou essai cedométrique, l'éprouvette est confinée dans le tube en Plexiglas, ce qui assure un déplacement radial nul en paroi du tube. Cependant, elle reste libre de se déformer dans le sens axial. Les parois internes du tube ont été préalablement graissées de façon à limiter les effets de frottement et par conséquence de frettage. Le capteur de déplacement permet à nouveau de suivre l'évolution du gonflement axial.

Pour le troisième essai, essai à volume constant, l'éprouvette ne peut pas se déformer macroscopiquement: elle est confinée dans le tube en Plexiglas et un piston poreux, positionné au sommet de l'éprouvette, bloque le gonflement macroscopique dans le sens axial. Une cellule de force à très faible déplacement (moins de $0,2 \mathrm{~mm}$ pour $1 \mathrm{MPa}$ ) reliée à ce piston permet d'accéder à la valeur de la pression de gonflement exercée par le milieu argileux dans le sens axial, au cours de son humidification.

Pour les trois essais, une balance de précision a été utilisée afin de mesurer la quantité d'eau absorbée par 
le milieu pendant toute la durée de l'expérience. À la fin de l'essai, les éprouvettes sont mesurées, pesées, puis placées à l'étuve. La valeur de la teneur en eau massique ainsi déterminée est ensuite comparée à celle obtenue par gammamétrie.
La figure 2 permet de visualiser schématiquement les différents types d'essai que nous avons réalisés. Nous avons réuni dans le tableau II, les caractéristiques initiales, dimensionnelles et pondérales, des éprouvettes utilisées.
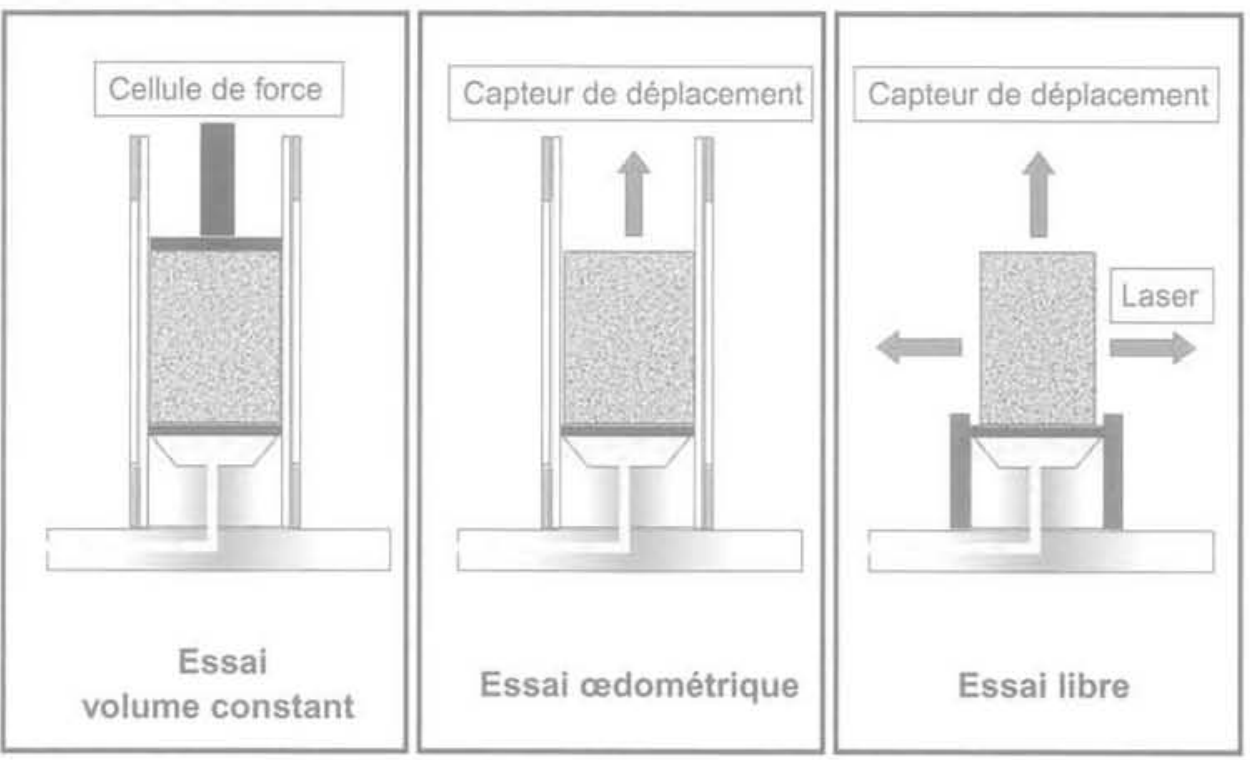

FG. 2 Schéma des différents types d'essais d'infiltration réalisés. Sketch of the different imbibition tests.

TABLEAU H Caractéristiques des différents essais réalisés. Characteristics of the different tests.

\begin{tabular}{l|c|c|c|c}
\multicolumn{1}{|c|}{ Types d'essai } & $\begin{array}{c}\text { Hauteur initiale } \\
(\mathrm{cm})\end{array}$ & $\begin{array}{c}\text { Diamètre initial } \\
(\mathrm{cm})\end{array}$ & $\left\langle p_{0}^{\text {gil }}\right\rangle\left(\mathrm{g} . \mathrm{cm}^{-3}\right)$ & $\left\langle\mathbf{w}^{\text {in's }}\right\rangle$ \\
\hline Volume constant & 8,06 & 6,0 & 1,52 & $25 \%$ \\
\hline E.dométrique & 8,06 & 6,0 & 1,50 & $25,1 \%$ \\
\hline Libre & 11,50 & 5,05 & 1,50 & $25,2 \%$ \\
\hline
\end{tabular}

\section{3}

\section{Gammamétrie double-source}

\section{1}

\section{Théorie}

Afin de suivre localement l'évolution de la teneur en eau et en solide dans un milieu poreux déformable, nous avons fait appel à la technique de gammamétrie double-source (Corey et al., 1971). Deux sources radioactives, Américium $\left(\mathrm{Am}^{2+1}\right)$ et Césium $\left(\mathrm{Cs}^{137}\right)$, émettant chacune des photons $\gamma$ d'énergies différentes (respectivement $60 \mathrm{keV}$ et $662 \mathrm{keV}$ ) sont placées dans un château de plomb de façon à créer un double faisceau colinéaire. Ces photons sont collectés, sélectionnés et comptabilisés par un détecteur couplé à un dispositif spectrométrique. Lorsque l'on place un échantillon de sol humide entre les sources et le détecteur, le faisceau $\gamma$ est atténué suivant une loi de type Beer-Lambert. Pour chacune des deux sources, cette atténuation s'écrit:

$$
\left\{\begin{array}{l}
N^{A m}=N_{0}^{A m} \exp \left[-\left(\mu_{s}^{A m} p_{s} \theta_{s}+\mu_{w}^{A m} \rho_{w} \theta_{s w}\right) x\right] \\
N^{C s}=N_{0}^{S} \exp \left[-\left(\mu_{s}^{C s} \rho_{s} \theta_{s}+\mu_{s w}^{C S} p_{w} \theta_{w}\right] x\right]
\end{array}\right.
$$

Les indices s et $w$ désignent respectivement le sol et l'eau, les $\mu$ représentent les coefficients d'absorption massique $\left(\mathrm{cm}^{2} \cdot \mathrm{g}^{-1}\right)$. $\mathrm{N}_{0}$ est le nombre de photons détectés "à vide») (c'est-à-dire en l'absence de l'échantillon de sol), $N$ est le nombre de photons détectés après traversée de l'éprouvette de sol humide, $x$ est l'épaisseur de l'éprouvette traversée par le faisceau $\gamma$. Nous noterons que l'atténuation due à l'air a été négligée. Le système ainsi écrit laisse apparaître deux inconnues: la teneur en eau volumique $\theta_{\omega}$ et la masse volumique sèche $\rho_{\mathrm{d}}$. Après résolution du système, nous obtenons:

$$
\begin{aligned}
& \rho_{d}=\rho_{s} \theta_{s}=\frac{\mu_{w}^{C s} \ln \left(N_{0}^{A m} / N^{A m}\right)-\mu_{w}^{A m} \ln \left(N_{0}^{C s} / N^{C s}\right)}{x\left(\mu_{s}^{A m} \mu_{w}^{C s}-\mu_{w}^{A m} \mu_{s}^{C s}\right)} \\
& \theta_{w}=\frac{\mu_{s}^{A m} \ln \left(N_{0}^{C s} / N^{C s}\right)-\mu_{s}^{C s} \ln \left(N_{0}^{A m} / N^{A m}\right)}{\rho_{w} \times\left(\mu_{s}^{A m} \mu_{w}^{C s}-\mu_{w}^{A m} \mu_{s}^{C s}\right)}
\end{aligned}
$$


Par ailleurs, il est aussi possible d'obtenir indirectement la teneur en eau massique, $w$ :

$$
w=\frac{\rho_{w} \theta_{w}}{\rho_{d}}=\frac{\mu_{s}^{A m} \ln \left(N_{0}^{C s} / N^{C s}\right)-\mu_{s}^{C s} \ln \left(N_{0}^{A m} / N^{A m}\right)}{\mu_{w}^{C s} \ln \left(N_{0}^{A m} / N^{A m}\right)-\mu_{w}^{A m} \ln \left(N_{0}^{C s} / N^{C s}\right)} .
$$

Par la suite, cette grandeur sera principalement utilisée (plutôt que $\theta_{w}$ ). En effet, w est le paramètre naturel (avec l'indice d'humidité) qui intervient dans l'écriture des équations de diffusion des sols gonflants, en suivant une description lagrangienne. Il peut très facilement être comparé avec les mesures faites par pesée (méthode par étuvage). De plus, comme le montre la relation (4), cette écriture permet de s'affranchir de la connaissance de l'épaisseur du milieu traversé par le faisceau $\gamma$. En général, les valeurs expérimentales sont déterminées avec une incertitude d'environ $\pm 0,04 \mathrm{~g} \cdot \mathrm{cm}^{-3}$ pour la masse volumique sèche $p_{\mathrm{d}}$. et d'environ $\pm 3 \%$ pour la teneur en eau massique.

Le système gammamétrique est disposé sur un banc de déplacement: il est alors possible de réaliser des mesures à n'importe quelle hauteur de l'éprouvette de sol. Nous présentons sur la figure 3 un schéma du dispositif expérimental utilisé.

\section{2}

\section{Détermination des caractéristiques hydrauliques}

Les mesures d'humidité et de masse volumique sèche obtenues par gammamétrie présentent un intérêt évident quant à la caractérisation du matériau en terme de diffusivité ou de conductivité hydraulique. Ces propriétés sont parfaitement bien définies dans le cas des milieux poreux non déformables (roches, bétons, sables...). En revanche, leur détermination s'avère plus complexe dans le cas des milieux déformables non saturés car il est indispensable de tenir compte de la nature gonflante du matériau. En 1968, Philip, Smiles et Rosenthal proposent une description lagrangienne des transferts hydriques dans les sols gonflants avec des conditions de Dirichlet:

$$
\frac{\partial w}{\partial t}=\frac{\partial}{\partial m}\left[D_{m}(w)\left(\frac{\partial w}{\partial m}\right)\right] \quad\left\{\begin{array}{l}
w=w^{\text {eit }} \text { pour } m=0 \text { et } t \geq 0 \\
w=w^{\text {tit }} \text { pour } m \rightarrow \infty \text { et } t \geq 0 \\
w=w^{\text {init }} \text { pour } m>0 \text { et } t=0
\end{array}\right.
$$

oủ $\mathrm{D}_{m}$ est la diffusivitê matérielle, m est la coordonnée matérielle qui lie le référentiel lagrangien au référentiel eulérien $\left(\mathrm{dm}=\rho_{\mathrm{d}} / \rho_{\mathrm{d}} \mathrm{dz}\right.$ ). Les effets de la gravité sont négligés. L'expression (5) peut être réécrite en lui appliquant une transformation de Boltzmann $\xi_{m}=m \sqrt{t}$ en prenant en compte la forte dépendance de la diffusivité $D_{m}$ avec $w$ :

$\frac{d w}{d \xi_{m}^{2}}=-\frac{1}{D_{m}(w)}\left[\frac{1}{2} \xi_{m} \frac{d w}{d \xi_{m}}+\frac{d D_{m}(w)}{d w}\left(\frac{d w}{d \xi_{m}}\right)\right]^{2}\left\{\begin{array}{l}w=w^{\text {ant }} \text { pour } \xi_{m}=0 \\ w=w^{m} \text { pour } \xi_{m} \rightarrow \infty\end{array}\right.$ ( 6.

L'hypothèse d'un milieu semi-infini n'est bien sûr valable que si le front d'humidification n'atteint pas la limite supérieure de l'éprouvette. La validation du modèle sera faite en pratique en traçant les évolutions de la teneur en eau massique en fonction de la variable de Boltzmann et en vérifiant que les courbes se superposent en un profil unique.

Remarque : afin de simplifier les écritures dans la suite de cet article, $m(z, t)$ pourra être remplacé par $m_{z}$, oủ $z$ est la hauteur issue du repère fixe.

\section{3}

\section{Identification avec un modèle explicite de diffusivité}

Si l'on se donne un modèle explicite de diffusivité, l'équation (6) peut être résolue par un algorithme classique de Runge-Kutta (d'ordre 4). Celui-ci est couplé à

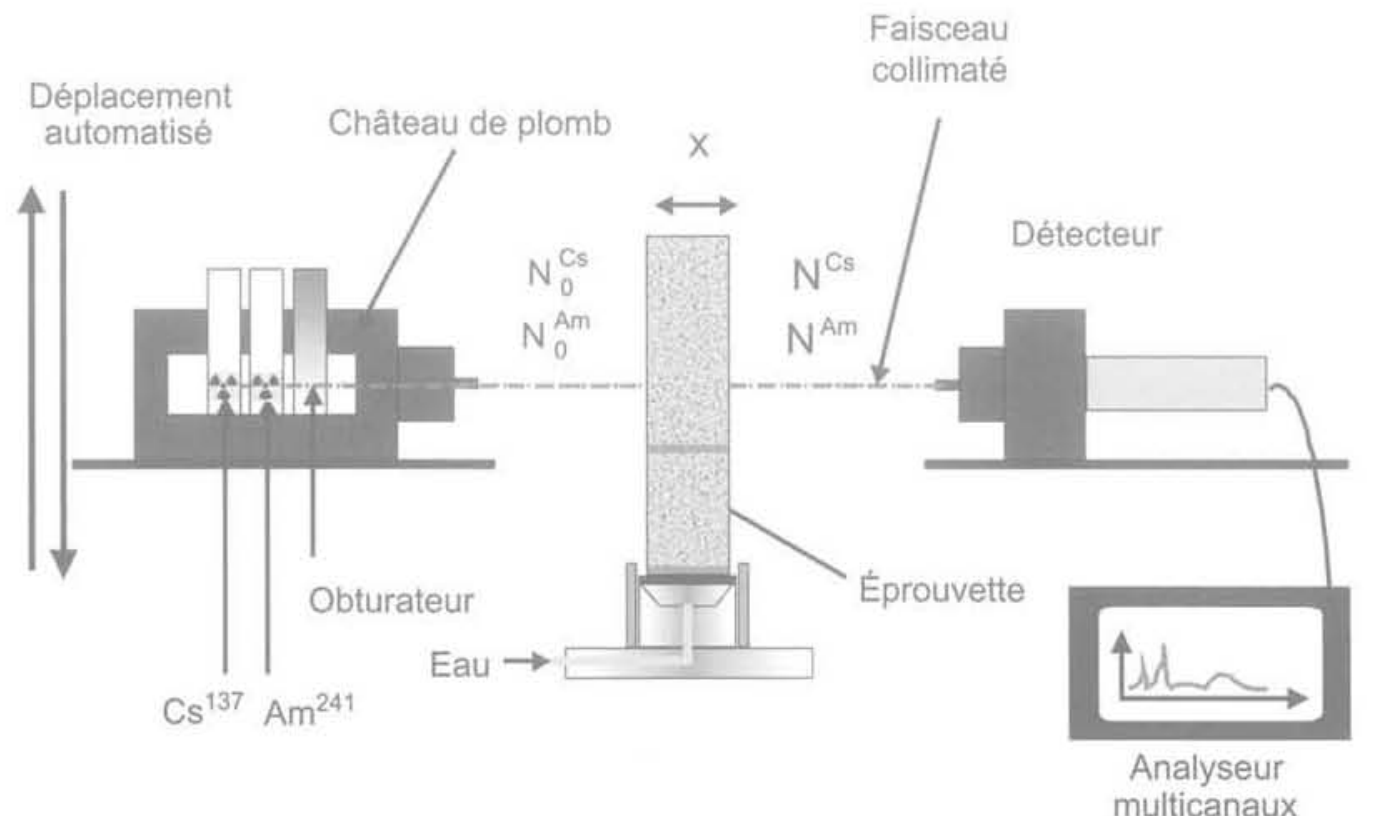


une méthode du tir de façon à remplacer la condition de teneur en eau imposée au sommet de l'éprouvette $\left(\xi_{\mathrm{m}} \rightarrow \infty\right)$ par une condition en terme de pente au bas de l'eprouvette $\left(\xi_{\mathrm{m}} \rightarrow 0\right)$ ). Une estimation correcte des paramètres du modèle de diffusivité choisi est obtenue en minimisant l'écart quadratique entre les valeurs des teneurs en eau simulées $w_{\text {um }}$ et les valeurs expérimentales $\mathrm{w}_{\text {exp }}$ (méthode des moindres carrés). La fonction objectif retenue, $f\left(w_{\text {sim }}, w_{\text {exp }}\right)=\sum_{\text {mesures }}\left(w_{\text {sim }}-w_{\text {exp }}\right)^{2}$, est minimisée selon un algorithme du Simplex (Press et al. 1986).

Dans notre programme, le modèle explicite de diffusivité matérielle choisi est une simple évolution exponentielle en fonction de la teneur en eau massique $w$ : $D_{-}(w)=a_{m} \exp \left(b_{n} w\right)$ où $a_{m}$ et $b_{-}$sont les paramètres à identifier. Le choix de ce modèle peut paraitre simpliste vu l'abondante littérature portant sur les modèles de conductivité ou de diffusivité hydraulique des sols. Ceppendant, ce modèle nous a semblé suffisant pour décrire les transferts đ'eau eu égard à la précision des résultats expérimentaux obtenus et, en anticipant sur la suite (Figs. 5, 10, 11, 13 et 14), compte tenu de la qualité acceptable des résultats d'identification finalement trouvés. Dans la pratique, nous identifions la pente de la courbe de Boltzmann en $\xi_{m} \rightarrow 0$. En revanche, nous imposons la valeur des teneurs en eau pour $\xi_{m} \rightarrow \infty$ et $\xi_{m} \rightarrow 0$. Pour $\xi_{\rightarrow} \rightarrow \infty$, w est fixée par rapport aux valeurs expérimentales observées avant de lancer l'infiltration $\left(\approx w^{i m}\right)$, elle sera notée $w_{k m}$.... La valeur de la teneur en eau pour $\xi_{m} \rightarrow 0, w_{\xi m}$. est évaluée en prolongeant de visu les courbes de Boltzmann expérimentales, et éventuellement réajustée pour qu'elle corresponde au mieux aux données expérimentales. La pertinence des valeurs imposées est finalement appréciée en superposant sur la représentation $\mathrm{w}=\mathrm{f}(\mathrm{t})$, les courbes simulées et les courbes expérimentales.

\section{4}

\section{Critiques de la technique d'identification}

En l'état actuel, la procédure d'identification de la diffusivité hydrique pose problème quant aux conditions aux limites imposées lors des essais. Parfaitement valables dans le cas des milieux non déformables, ces conditions ne rendent pas tout à fait compte de la réalité expérimentale. En effet, au début de l'imbibition. l'eau commence à humidifier le bas de l'éprouvette mais en raison du gonflement simultané du milieu, la teneur en eau massique varie conjointement. II n'est donc pas possible d'imposer une teneur en eau constante durant l'essai par simple mise en contact avec une nappe d'eau dans le cas d'un milieu poreux déformable.

Pour peu que le flux d'eau infiltré soit connu (ce qui est le cas pour nos essais), il est donc préférable d'effectuer l'identification de la diffusivité matérielle en supposant des conditions de type Neumann let non de type Dirichlet). Le caractère non linéaire de l'équation de diffusion et la condition non homogène imposée ne nous permettent plus de résoudre simplement le problème par transformation de Boltzmann comme auparavant. II est alors nécessaire de recourir à une résolution numérique de l'équation aux dérivées partielles discrétisée, assortie de ses conditions aux limites (de type Neumann). La procédure d'identification de la diffusivité matérielle peut également être envisagée par une méthode des moindres carrés couplèe à une optimisation par un algorithme du Simplex.

Cependant, l'utilisation d'une méthode de résolution numérique alourdit considérablement la technique d'identification, puisque la solution du problème doit être recalculée numériquement à chaque étape. C'est pourquoi, nous avons privilégié l'utilisation de la technique d'identification, couplée aux conditions de Dirichlet. Un programme numérique intégrant des conditions de Neumann nous a malgré tout permis de contrôler dans le sens direct la validité des ordres de grandeur obtenus. Nous le verrons dans la suite de cet article.

\section{4}

\section{Résultats et discussions}

\section{1}

\section{Validation sur un matériau non déformable}

Toute la méthodologie a été préalablement testée sur un matériau de référence non déformable, qui se prête bien aux mesures par gammamétrie: le béton cellulaire. Nous avons ainsi réalisé un essai d'infiltration sur un échantillon de hauteur $20 \mathrm{~cm}$ et d'épaisseur $5,2 \mathrm{~cm}$. La porosité de l'échantillon $(71 \%)$ et sa masse volumique sèche $\left(\rho_{\mathrm{r}}=0,52 \mathrm{~g} \cdot \mathrm{cm}^{-3}\right)$ ont été mesurées par méthode gravimétrique. Nous présentons sur la figure 5 , les courbes d'humidification obtenues sur ce matériau pour quatre hauteurs différentes. La représentation de $w$ en fonction de la variable de Boltzmann classique $(\mathrm{z} / \mathrm{Vt})$ permet d'obtenir une courbe expérimentale unique, conformément à ce qui est décrit dans la théorie (Fig. 4). Cela signifie physiquement que les phénomènes dans ce type de milieu sont effectivement purement diffusifs et que l'hypothèse consistant à négliger les forces de gravité est pertinente (la faible hauteur de l'échantillon permettait déjà de supposer cette approximation). En utilisant ensuite un modèle explicite de diffusivité, $D(w)=a \exp (b w)$, notre programme d'identification nous permet d'obtenir a = $2.75 .10^{-10} \mathrm{~m}^{2} \cdot \mathrm{s}^{-1}, \mathrm{~b}=9,74$ pour la hauteur $\mathrm{z}=12 \mathrm{~cm}$. Sur la figure 4, la courbe en trait plein permet de juger de la qualité de notre identification. Nous vérifions ensuite sur la figure 5 que le jeu de paramètres identifiés permet effectivement de retrouver l'allure générale de la courbe d'infiltration à la hauteur considérée. Avec ce même jeu de paramètres, nous parvenons également à simuler très correctement les réponses aux hauteurs $\mathrm{z}=4,8,16 \mathrm{~cm}$.

Ce travail préalable sur un matériau de référence a permis de mettre en évidence la pertinence du dispositif expérimental, et du traitement des données qui s'ensuit. Qu'advient-il maintenant lorsque l'on travaille sur un matériau déformable?

\section{9}

\section{Résultats sur le matériau gonflant}

\section{Lagif \\ Coefficients d'absorption massique}

Les coefficients d'absorption du rayonnement $\gamma$ de l'eau sont obtenus en utilisant différentes éprouvettes 


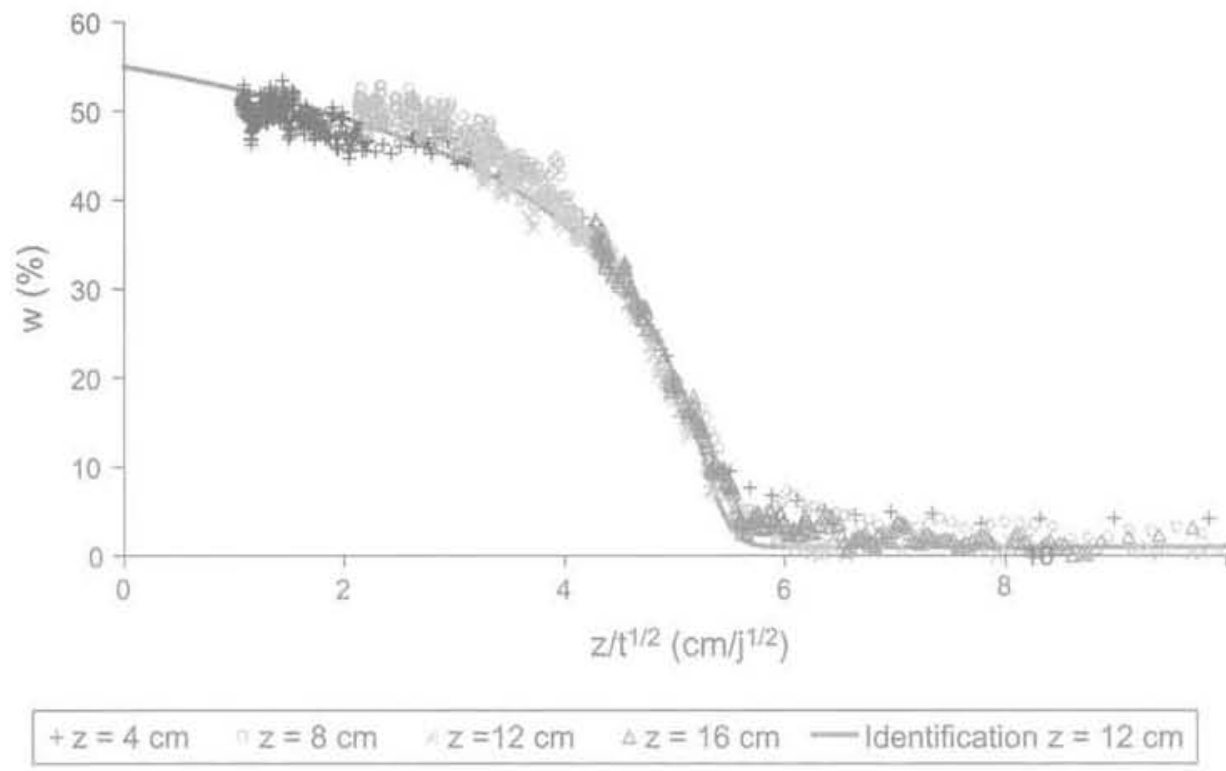

FG. 4 Évolution de la teneur en eau massique en fonction de la variable de Boltzmann; comparaison des résultats expérimentaux et de la courbe identifiée (béton cellulaire).

Evolution of the water content versus the Boltzmann variable; comparison of experimental and identified results (cellular concrete).

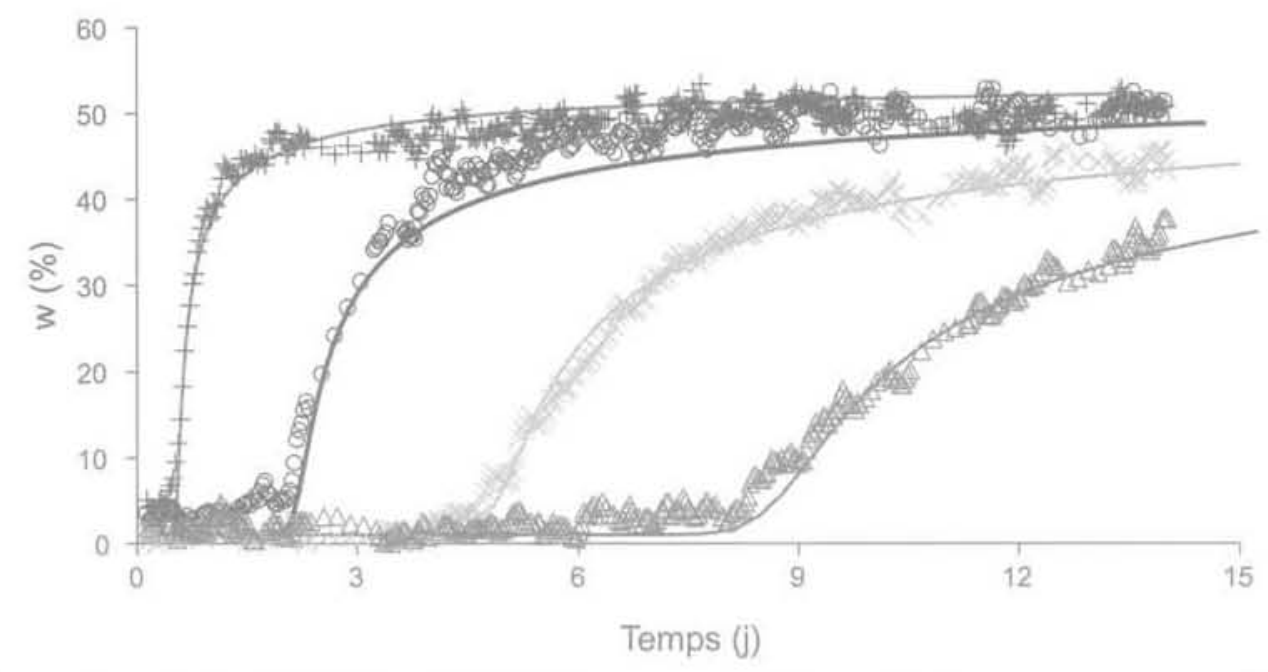

\begin{tabular}{|c|c|c|c|}
\hline $\begin{array}{l}+z=4 \mathrm{~cm} \\
- \text { Simulation } z=4 \mathrm{~cm}\end{array}$ & $\begin{array}{l}\text {. } z=8 \mathrm{~cm} \\
\text { Simulation } z=8 \mathrm{~cm}\end{array}$ & $\begin{array}{l}z=12 \mathrm{~cm} \\
\text { Identification } z=12 \mathrm{~cm}\end{array}$ & $\begin{array}{l}\Delta z=16 \mathrm{~cm} \\
\longrightarrow \text { Simulation } z=16 \mathrm{~cm}\end{array}$ \\
\hline
\end{tabular}

FG.5 Courbes d'imbibition obtenues sur du béton cellulaire, avec les résultats de l'ídentification. Imbibition curves for the cellular concrete, with the results of identification.

calibrées (utilisées en spectroscopie) d'èpaisseur 2 et $5 \mathrm{~cm}$, remplies d'eau distillée et combinées afin d'obtenir successivement des lames d'eau de 2, 4, 9, 14 , $19 \mathrm{~cm}$, entre les sources et le détecteur. Les valeurs ainsi déterminées (Tableau III) correspondent assez bien à celles fournies dans la littérature $(0,2036$ et $0,0858 \mathrm{~cm}^{2} \cdot \mathrm{g}^{-1}$ pour Gardner et al., 1972; 0,2040 et $0,0838 \mathrm{~cm}^{2} \cdot \mathrm{g}^{-1}$ pour Angulo, 1989; 0,2042 et $0,0820 \mathrm{~cm}^{2} \cdot \mathrm{g}^{-1}$ pour Barataud et al., 1999).

Le protocole utilisé dans le cas de l'eau n'est malheureusement pas envisageable pour déterminer les coefficients intrinsèques à la fraction solide du sol gonflant. En effet, nos premières investigations (Tabani, 1999) ainsi que d'autres travaux (Barataud et al., 1999) déconseillent une détermination des coefficients sur poudre sèche. Sans que l'on puisse vraiment expliquer toutes les raisons physiques, ces coefficients ne permettent pas par la suite de redonner des profils d'humidité et de densité corrects pour une éprouvette compactée, constituée de cette même poudre. Nous avons donc adopté une autre méthodologie pour ce sol, consistant à calculer directement les coefficients sur les éprouvettes compactées avant infiltration. Nous utilisons l'équation suivante ("représente la source Am ou bien Cs):

$$
\mu_{\mathrm{s}}^{*}=\frac{1}{\mathrm{I}} \sum_{i}^{1} \frac{1}{\mathrm{x} \cdot\left\langle\rho_{\mathrm{d}}^{\mathrm{ini}}\right\rangle} \cdot \ln \left(\frac{\left(\mathrm{N}_{0}\right)_{i}^{*}}{(\mathrm{~N})_{i}^{*}}\right)-\mu_{\mathrm{w}}^{*} \cdot\left\langle\mathrm{w}^{\mathrm{ini} i}\right\rangle
$$




\begin{tabular}{|c|c|c|c|}
\hline Eau & $\begin{array}{c}\text { Sol } \\
\text { (libre) }\end{array}$ & $\begin{array}{c}\text { Sol } \\
\text { (cedométrique) }\end{array}$ & $\begin{array}{c}\text { Sol } \\
\text { (volume constant) }\end{array}$ \\
\hline$\mu_{w}^{\mathrm{Am}}=0,2040 \pm 0,0003$ & $\mu_{s}^{\lambda m}=0,318 \pm 0,002$ & $\mu_{\mathrm{s}}^{\mathrm{Am}}=0,325 \pm 0,002$ & $\mu_{s}^{A m}=0,326 \pm 0,002$ \\
\hline$\mu_{w}^{\circ}=0,0807 \pm 0,0001$ & $\mu_{s}^{\infty}=0,0693 \pm 0,0006$ & $\mu_{s}=0,0833 \pm 0,0006$ & $\mu_{s}^{C s}=0,0780 \pm 0,0006$ \\
\hline
\end{tabular}

où I représente le nombre de points constituant le profil et $i$ correspond au rang du point scruté. Le tableau III récapitule les coefficients utilisés pour les différents essais.

Celui-ci met en évidence un problème, qui n'est apparemment pas abordé dans la littérature, si ce n'est par Barataud et al. (1999) et Rolland (2002). Les couples de coefficients obtenus ne sont jamais tout à fait identiques d'une éprouvette à une autre, malgré le fait qu'elles aient quasiment des masses volumiques et des teneurs en eau massiques identiques. Nous émettons deux hypothèses pour expliquer cette constatation: - les coefficients d'absorption massique ne sont pas mesurés avec une précision suffisante. Il est vrai qu'ils sont déterminés de manière indirecte et qu'un certain nombre de données entrent en jeu (pesée, fluctuations gammamétriques, temps de comptage, étalonnage);
- les coefficients d'absorption de l'eau ne sont pas parfaitement définis suivant son état dans le sol (eau libre, eau liée...).

Pour un mème essai, cela se traduit à la fin de l'essai, par un léger décalage entre les grandeurs mesurées par gammamétrie et celles obtenues par étuvage (Tableau IV). Cet écart est plus important dans le cas de l'essai libre, car la quantité d'eau imbibée est plus importante elle aussi.

\section{0 \\ Courbes d'humidification et de gonflement}

Nous présentons sur les figures 6 (essai libre), 7 (essai cedométrique), 8 (essai à volume constant), l'évolution de la masse volumique sèche en fonction du temps à deux hauteurs différentes $(2,5 \mathrm{~cm}$ et $5,5 \mathrm{~cm})$.

TABLEAUN Comparaison des teneurs en eau massiques finales obtenues par gammamétrie et par pesée. Comparison between water contents determined by gamma technique and by weighting.

\begin{tabular}{|c|c|c|c|}
\hline & Essai libre & Essai cedométrique & Essai volume constant \\
\hline Gammamétrie & $\left\langle W^{\text {thin }}\right\rangle=43,9 \%$ & $\left\langle\mathrm{w}^{\operatorname{lin}}\right\rangle=42,6 \%$ & $\left\langle W^{f m}\right\rangle=32,2 \%$ \\
\hline Pesée & $\left\langle w^{10}\right\rangle=48,1 \%$ & $\left\langle w^{\operatorname{lin}}\right\rangle=40 \%$ & $\left\langle w^{n i l i}\right\rangle=30,8 \%$ \\
\hline
\end{tabular}

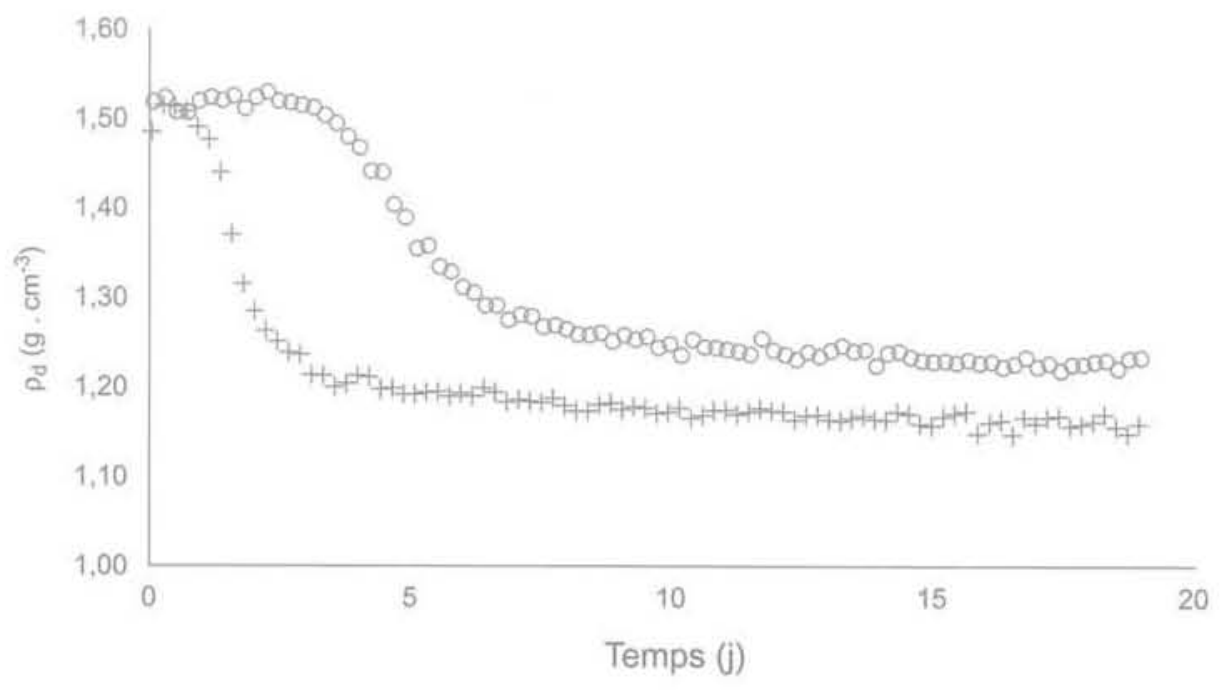

$$
+z=2,5 \mathrm{~cm} \circ z=5,5 \mathrm{~cm}
$$

FG.6 Évolution de la masse volumique sèche de l'éprouvette de sol pour deux hauteurs différentes dans le cas de l'essai libre.

Variation of the bulk density of our soil sample for two different heights in the case of the free test. 


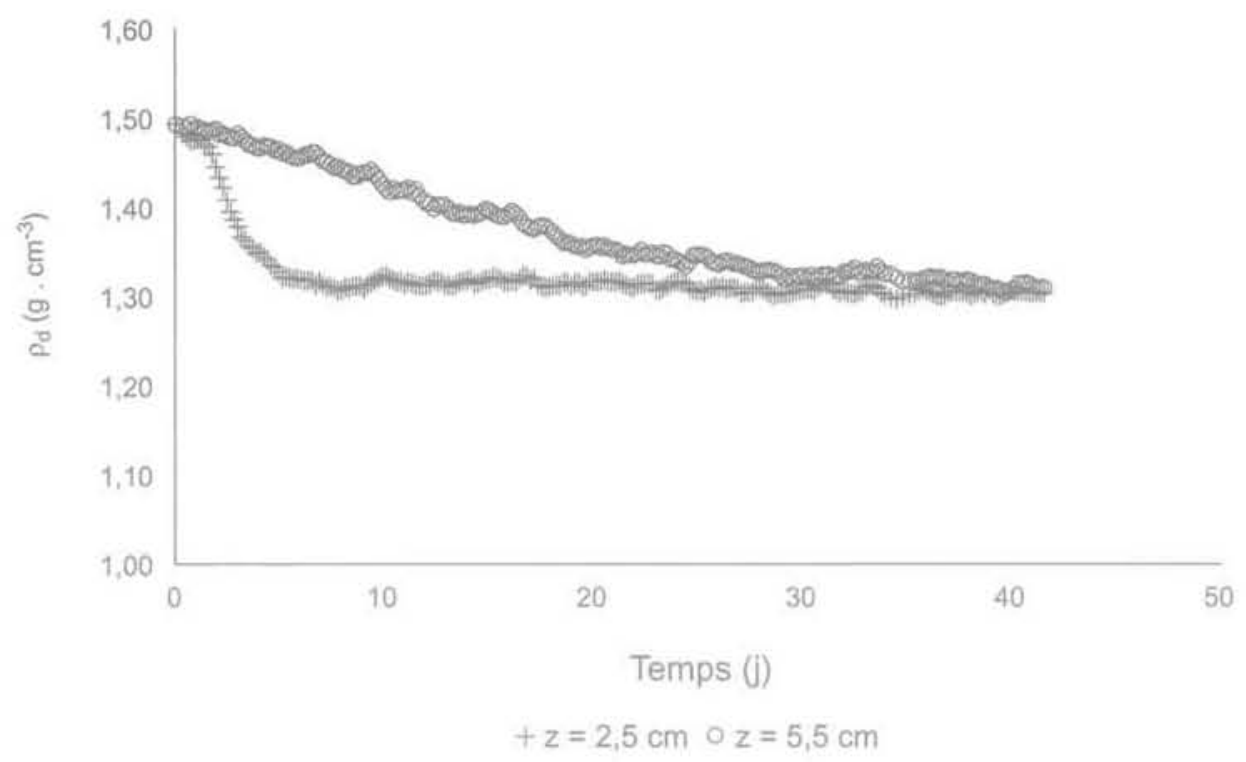

FG, 7 Évolution de la masse volumique sèche de l'éprouvette de sol pour deux hauteurs différentes dans le cas de l'essai œedométrique.

Variation of the bulk density of the soil sample for two different heights in the case of the oedometric test.

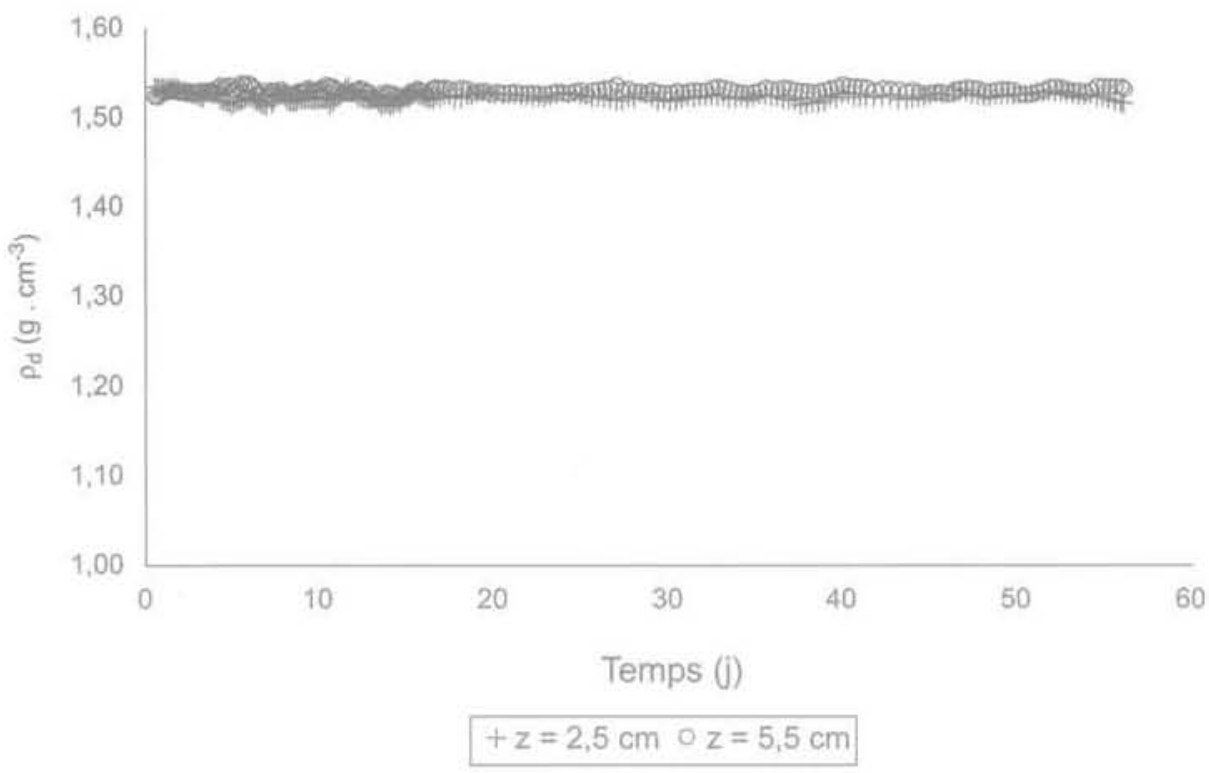

FG. 8 Évolution de la masse volumique sèche de l'éprouvette de sol pour deux hauteurs différentes dans le cas de l'essai à volume constant.

Variation of the bulk density of the soil sample for two different heights in the case of the constant volume test.

Rappelons que pour les 3 essais, l'état initial du matériau est sensiblement le même (Tableau II). Ces trois courbes traduisent le gonflement local des échantillons suite à l'imbibition d'eau. Une comparaison entre les courbes 6 et 7 montre clairement que le développement des contraintes mécaniques, résultant du gonflement du matériau et de son confinement extérieur, ralentit considérablement le transfert hydrique à l'intérieur du milieu. En effet, pour l'essai œedométrique, la courbe à la hauteur $\mathrm{z}=5,5 \mathrm{~cm}$, montre qu'il faut au moins deux fois plus de temps que pour l'essai libre pour atteindre un état d'équilibre. Ceci est encore plus vrai pour l'essai à volume constant pour lequel la cinétique d'infiltration varie très lentement. Conjointement, la figure 8 relative à l'essai à volume constant permet de vérifier que le gonflement local reste très faible, sans toutefois être nul, alors que, dans le même temps (c'est-à-dire environ deux mois), la teneur en eau massique de l'échantillon est passée de $25 \%$ à près de $30 \%$ (Tableau IV).

Les figures $9,10,11$ illustrent respectivement l'évolution de la teneur en eau massique en fonction de la variable de Boltzmann. L'unicité des courbes est relativement bien respectée dans le cas des essais œdométrique et à volume constant. Elle est, en revanche, moins bonne pour l'essai libre pour des hauteurs situées en bas de l'éprouvette. Cela peut s'expliquer de différentes manières. Comme nous l'avions précisé précédemment, les conditions aux limites sont mal assurées en bas de l'éprouvette: l'écoulement dans cette zone ne peut vraisemblablement plus être considéré 


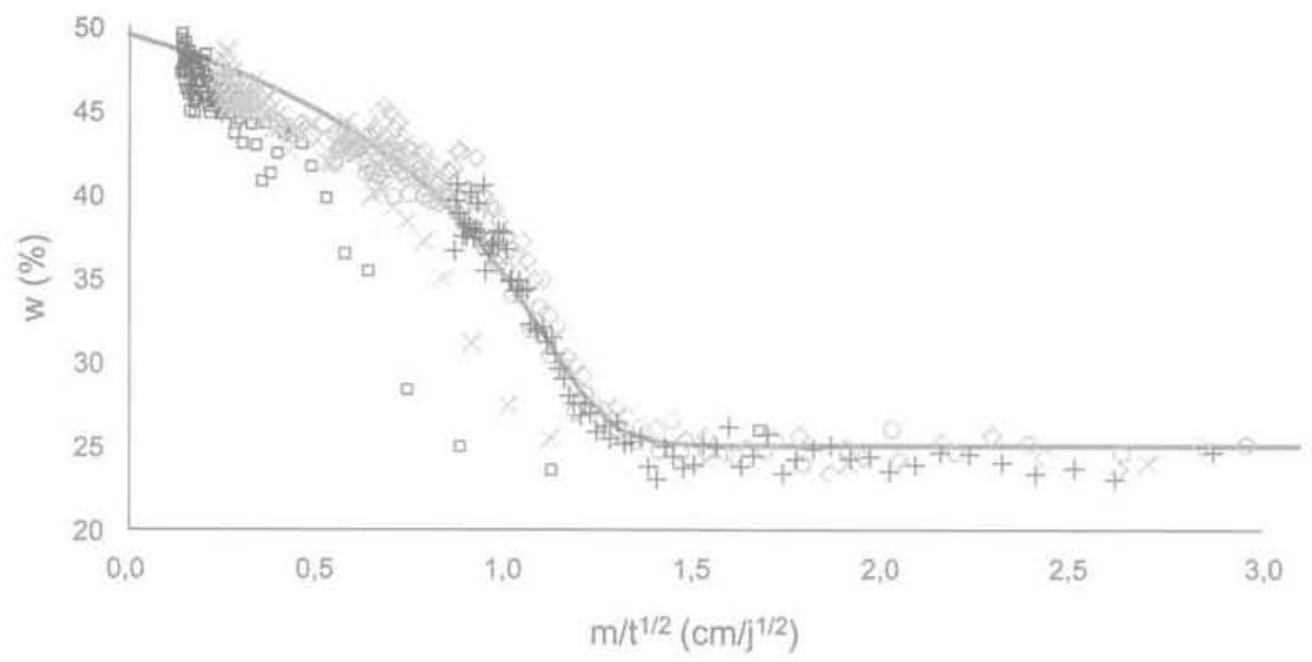

$\begin{array}{lll}\mathrm{m}(\mathrm{z}=1,5 \mathrm{~cm}, \mathrm{t}) & \mathrm{m}(\mathrm{z}=2,5 \mathrm{~cm}, \mathrm{t}) & \mathrm{m}(\mathrm{z}=5,5 \mathrm{~cm}, \mathrm{t}) \\ \mathrm{m}(\mathrm{z}=6,5 \mathrm{~cm}, \mathrm{t}) & +\mathrm{m}(\mathrm{z}=8,5 \mathrm{~cm}, \mathrm{t}) & \text { - Identification moyenne }\end{array}$

FG.9 Évolution de la teneur en eau massique en fonction de la variable de Boltzmann matérielle dans le cas de l'essai libre et courbe identifiée.

Boltzmann representation in the case of the free test and identified curve (material coordinates).

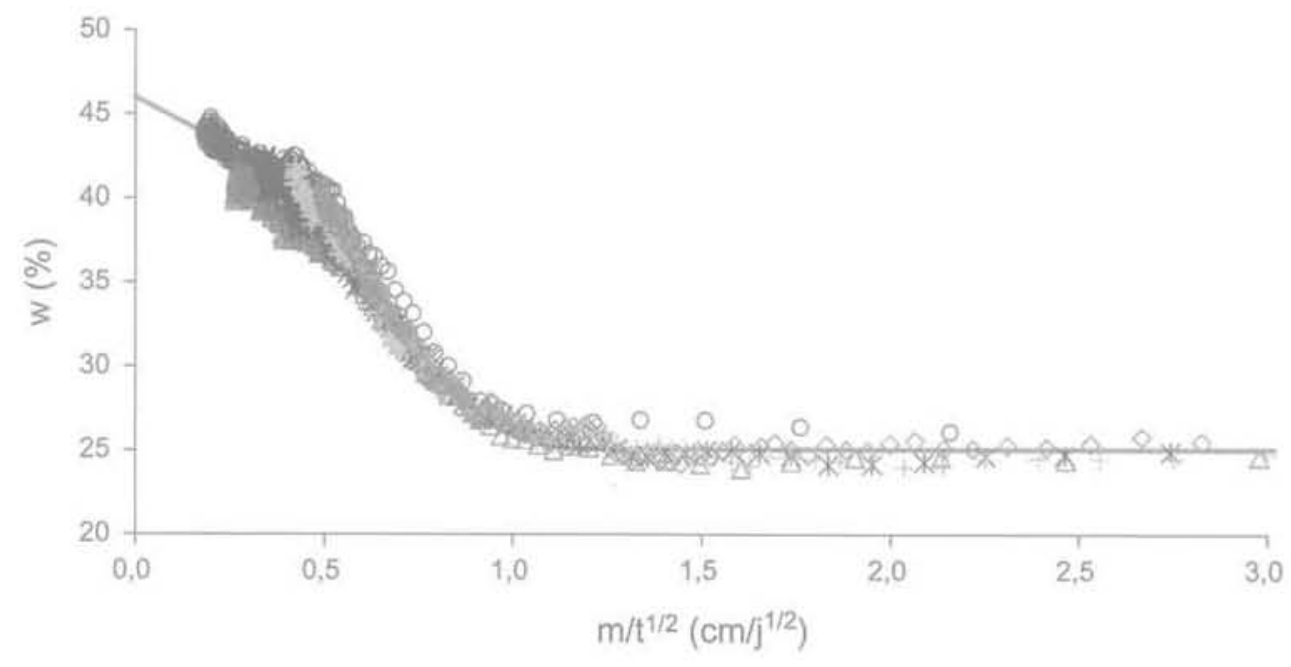

$$
\begin{array}{rll}
\circ m(z=2,5 \mathrm{~cm}, t) & \Delta m(z=3,5 \mathrm{~cm}, \mathrm{t}) & * m(z=4,5 \mathrm{~cm}, \mathrm{t}) \\
+m(z=5,5 \mathrm{~cm}, \mathrm{t}) & \Delta \mathrm{m}(\mathrm{z}=6,5 \mathrm{~cm}, \mathrm{t}) & \text { - Identification moyenne }
\end{array}
$$

FiG. 10 Évolution de la teneur en eau massique en fonction de la variable de Boltzmann matérielle dans le cas de l'essai œdométrique et courbe identifiée.

Boltzmann representation in the case of the oedometric test and identified curve (material coordinates). 


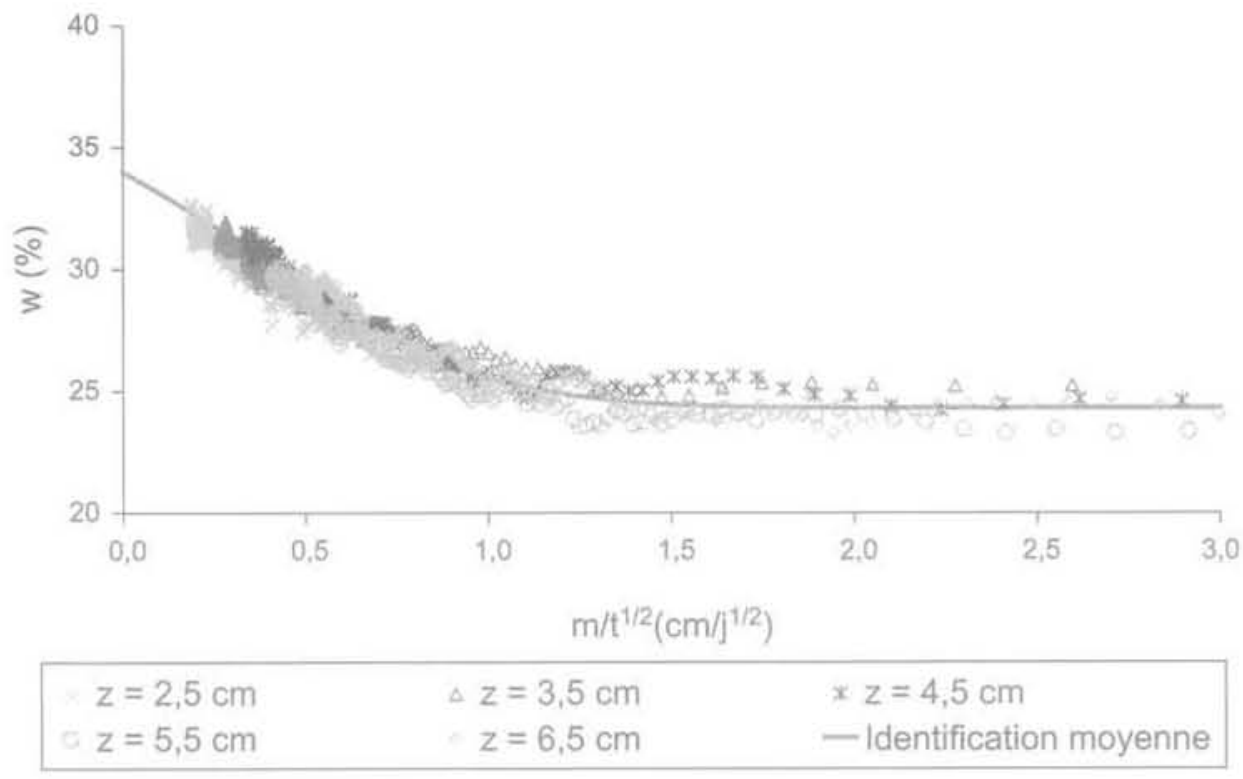

FIG. 11 Évolution de la teneur en eau massique en fonction de la variable de Boltzmann matérielle dans le cas de I'essai à volume constant et courbe identifiée.

Boltzmann representation in the case of the volume constant test and identified curve (material coordinates).

TABLEAUV Récapitulatif des paramètres identifiés à différentes hauteurs de l'éprouvette de sol (essai cedométrique). Summary of the different identified parameters at different levels of the soil specimen (oedometric test).

\begin{tabular}{|c|c|c|c|c|c|}
\hline $\begin{array}{l}\text { Coordonnées matérielies } \\
\text { (cm) }\end{array}$ & $m_{1 s}$ & $m_{35}$ & $m_{40.0}$ & $\mathrm{~m}_{25}$ & $m_{i a}$ \\
\hline$a_{m}\left(m^{2} \cdot s^{-1}\right)$ & $1,88.10^{-33}$ & $3,55,10^{-12}$ & $1,66.10^{-11}$ & $9,79.10^{-12}$ & $2,85.10^{-13}$ \\
\hline$b_{10}$ & 19,3 & 11.1 & 6,6 & 14,8 & 17,7 \\
\hline Pente $\left(\mathrm{s}^{1 / 2} \cdot \mathrm{m}^{-1}\right)$ & $-2,33.10^{-2}$ & $-4,05.10^{-2}$ & $-5,92.10^{-2}$ & $-3,09,10^{-2}$ & $-2,73.10^{-2}$ \\
\hline$\left[w_{t+1+2} ; w_{t+1}=0\right]$ & {$[45: 26]$} & {$[45 ; 25]$} & {$[50 ; 24,5]$} & $\lfloor 45 ; 25]$ & {$[45 ; 25]$} \\
\hline
\end{tabular}

comme unidimensionnel. De plus, au cours de l'imbibition, nous avons relevé l'apparition de petites fissures, qui ne sont pas prises en compte dans la modélisation des transferts.

\section{4h.}

\section{Identification de la diffusivité hydraulique}

Afin d'accéder à la diffusivité matérielle du matériau, tout en tenant compte des imperfections visibles sur les représentations de Boltzmann, nous avons identifié les paramètres $\mathrm{a}_{m}$ et $\mathrm{b}_{\mathrm{m}}$ sur la gamme de teneurs en eau explorée, pour des hauteurs éloignées des limites haute et basse de l'échantillon. Nous donnons par exemple les valeurs obtenues dans le cas de l'essai œedométrique dans le tableau V. Les paramètres étant assez différents les uns des autres pour les différentes hauteurs considérées, nous avons tracé sur la figure 12, les courbes de diffusivité en fonction de la gamme de teneur en eau explorée. Toutes conservent le même ordre de grandeur mais les valeurs à l'état initial sont assez dispersées. Cela laisse penser que le concept de diffusivité matérielle n'est pas parfaitement adapté au problème, ou bien que le modèle exponentiel est trop simpliste pour décrire le processus diffusif avec gonflement. Nous en rediscuterons au paragraphe suivant. 


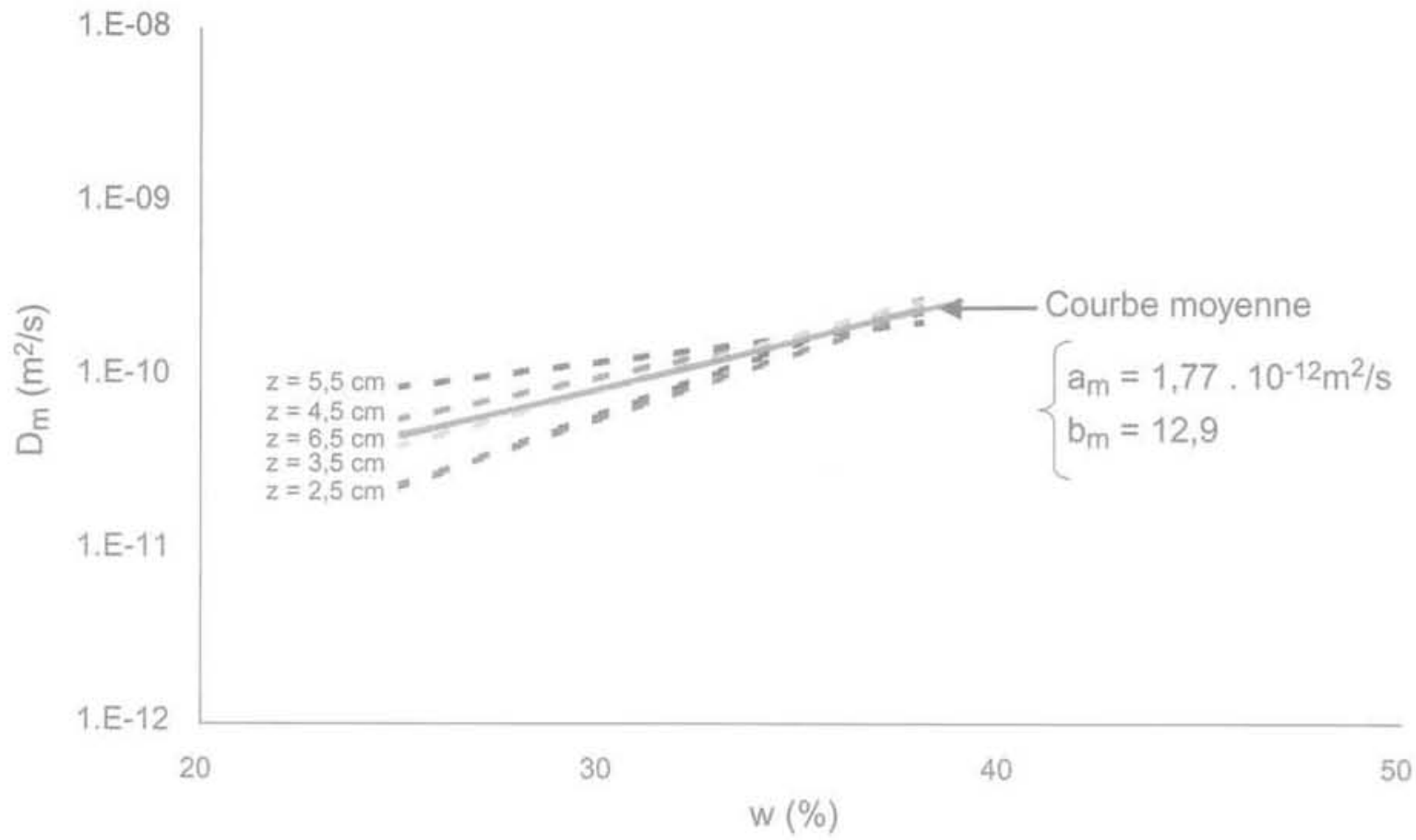

FG.12 Courbes de diffusivité en fonction de la teneur en eau pour différents points de mesure (essai œdométrique).

Diffusivity curves versus water content at different measurement points (oedometric test),

La procédure utilisée ensuite consiste à substituer à toutes ces courbes de diffusivité une courbe movenne donnant un poids égal à toutes les hauteurs examinées:

$$
\left\langle D_{m}(w)\right\rangle=\frac{1}{5} \sum_{i=1}^{5} D_{m}(w, m)
$$

où i désigne le point de mesure réalisé pour la coordonnée matérielle $\mathrm{m}$. On ajuste à nouveau sur cette courbe moyenne un modèle exponentiel $\left(\left\langle\mathrm{a}_{\mathrm{m}}\right\rangle=\right.$ $\left.1,77.10^{-12} \mathrm{~m}^{2} \cdot \mathrm{s}^{-1},\left\langle\mathrm{~b}_{\mathrm{n}}\right\rangle=12,9\right)$. La figure 13 permet de contrôler la validite du jeu de paramètres moyens obtenu: l'allure des courbes d'imbibition simulées correspond plutôt bien aux courbes expérimentales. La figure 14 permet de comparer les courbes expérimentales avec les courbes calculées numériquement, en imposant le flux d'eau infiltrée fourni par la balance (conditions de Neumann) et en injectant simplement le jeu de paramètres moyen précédent. Des différences sont évidemment visibles parce que le programme numérique utilisé n'est qu'une simple résolution de l'équation (5) sans identification et parce que le flux expérimental imposé est imprécis aux temps courts et reste bruité tout au long de l'essai. Cependant, on peut

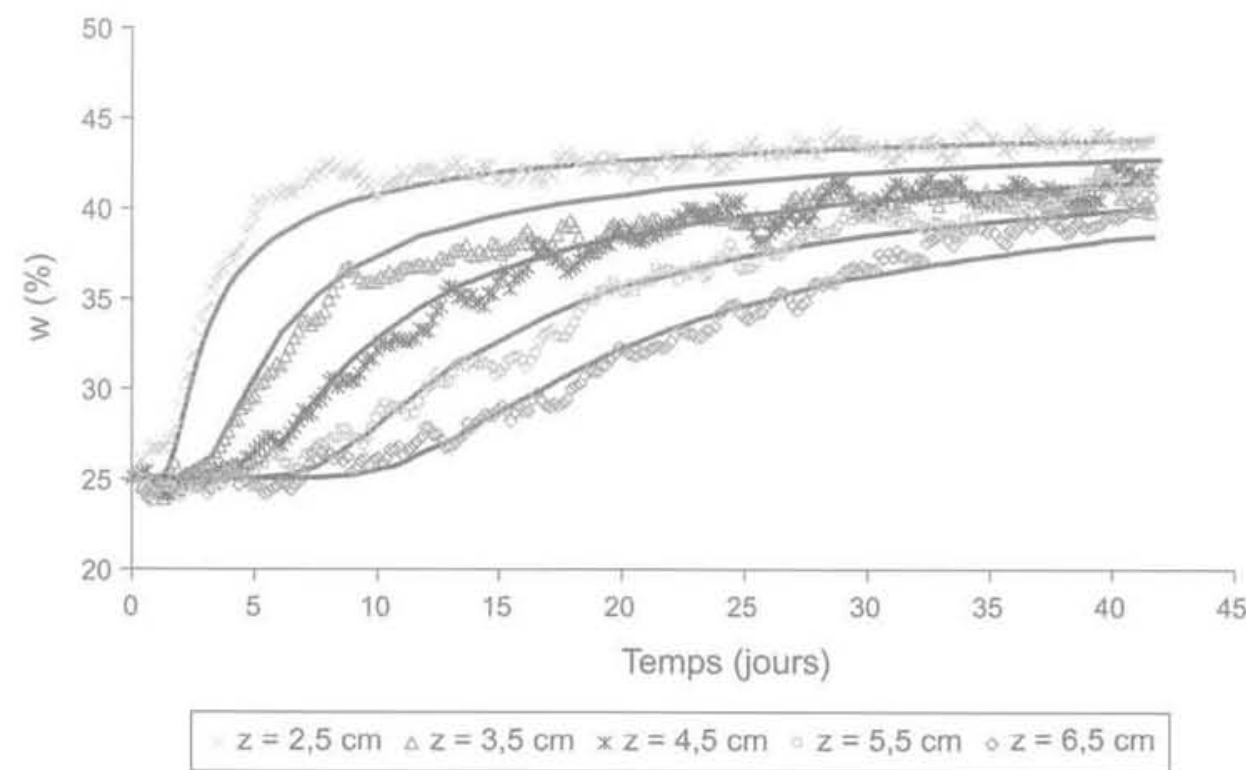

FG. 13 Courbes d'humidification dans le cas de l'essai œedométrique et résultat de l'identification. Imbibition curves in the case of oedometric test and result of the identification. 


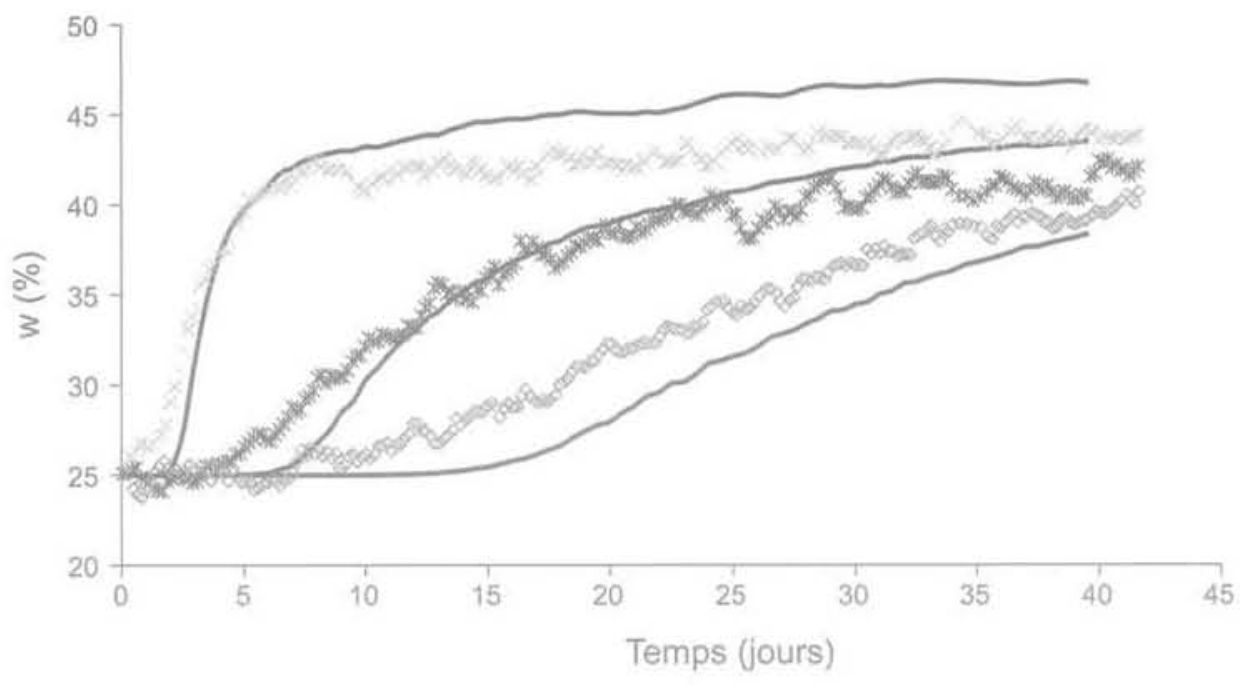

$z=2,5 \mathrm{~cm} \times z=4,5 \mathrm{~cm} \odot z=6,5 \mathrm{~cm}$

FG. 14 Courbes d'humidification dans le cas de l'essai œedométrique et résultat direct de l'identification précédente avec des conditions de Neumann.

imbibition curves in the case of oedometric test and direct result of the previous identification with Neumann conditions.

noter que les ordres de grandeur résultants demeurent acceptables.

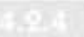

\section{Comparaison des trois essais}

L'opération a été reconduite pour les deux autres expériences. Cela nous permet de comparer les résultats en terme de diffusivité matérielle (Fig. 15). Mathématiquement, nous rappelons que la diffusivité matérielle est définie par le produit de trois termes (Angulo, 1989) :

$$
D_{m}(w)=k_{w s s}\left(\frac{\rho_{w} \rho_{d}}{\rho_{s}^{2}}\right)\left(\frac{d \psi_{w}}{d w}\right)
$$

Le premier terme représente la conductivité hydraulique (par rapport à la phase solide) au sens de Darcy, le deuxième caractérise l'état de déformation du milieu, et le troisième correspond à la pente de la courbe de rétention. La littérature montre que si la teneur en eau augmente, le premier et le troisième terme peuvent varier de plusieurs ordres de grandeur, avec un comportement opposé: la conductivité croît tandis que la courbe de rétention décroit. Le deuxième terme diminue lui aussi, mais relativement peu par rapport aux deux autres. Il ne joue donc pas un rôle déterminant dans l'évolution de la diffusivité. Pour un même matériau, le concept de diffusivité matérielle, qui doit tenir compte de l'évolution de ces trois termes, est en théorie une propriété intrinsèque. Pour nos essais, nous aurions donc dû obtenir une superposition des diffusivités autour d'une courbe unique. Cela n'est manifestement pas le cas au regard de la figure 15 , ce qui mérite une explication.

Intéressons-nous tout d'abord aux essais œedométrique et libre. Nous observons que si les valeurs des

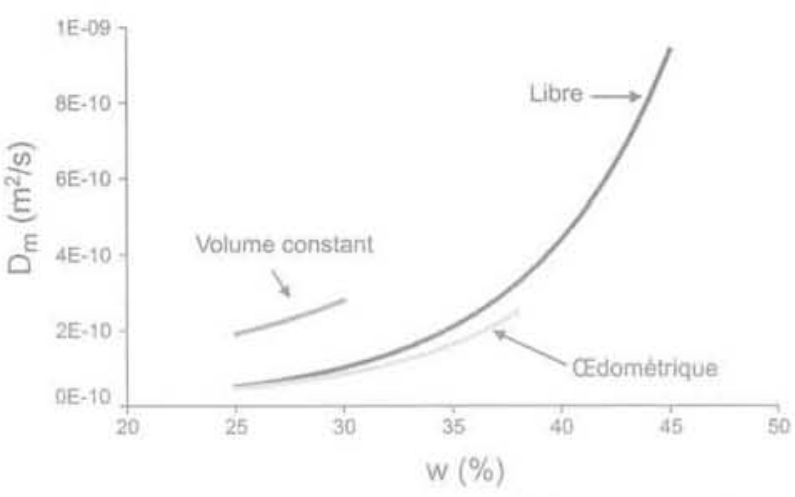

fıG. 15 Comparaison des différents essais en terme de diffusivité matérielle.

Comparison of the different tests in terms of material diffusivity.

diffusivitês sont du même ordre de grandeur à l'état initial, elles se différencient assez nettement, suivant le confinement, aux fortes teneurs en eau.

En évoquant une structure multi-échelle du matériau (feuillets, particules, agrégats), se traduisant par différents niveaux de porosité (microporosité, mésoporosité, macroporosité), il est possible d'imaginer, compte tenu des conditions expérimentales, que les phénomènes de gonflement, d'origine microscopique, entraînent une augmentation de la microporosité (voire de la mésoporosité) au détriment de la macroporosité. En effet, l'eau qui pénètre dans l'éprouvette va s'infiltrer d'abord dans l'espace interagrégat, et progressivement dans les espaces interparticulaires et interfoliaires. Cela a pour conséquence:

- un écartement des feuillets, donc un gonflement des particules: 
- une répulsion des particules argileuses, expliquée à partir de la théorie de la double couche (Mitchell, 1993).

Dans le cas de l'essai œdométrique, le gonflement macroscopique radial est nul, les espaces interfoliaires et interparticulaires d'une même section vont donc s'accroitre au détriment de l'espace interagrégat. Ce mécanisme se traduit évidemment, à l'échelle macroscopique, par une circulation de l'eau rendue plus difficile pour l'essai œedométrique en fonction du gonflement. La courbe de diffusivité matérielle a donc une amplitude moins prononcée et explore une gamme de teneur en eau plus restreinte que dans le cas de l'essai libre. Ce phénomène de redistribution de la porosité a déjà été observé et interprété identiquement par d'autres auteurs Garnier et al. (1998), Tabani (1999), Villar et Lloret (2001). Nous l'avons schématisé sur la figure 16.

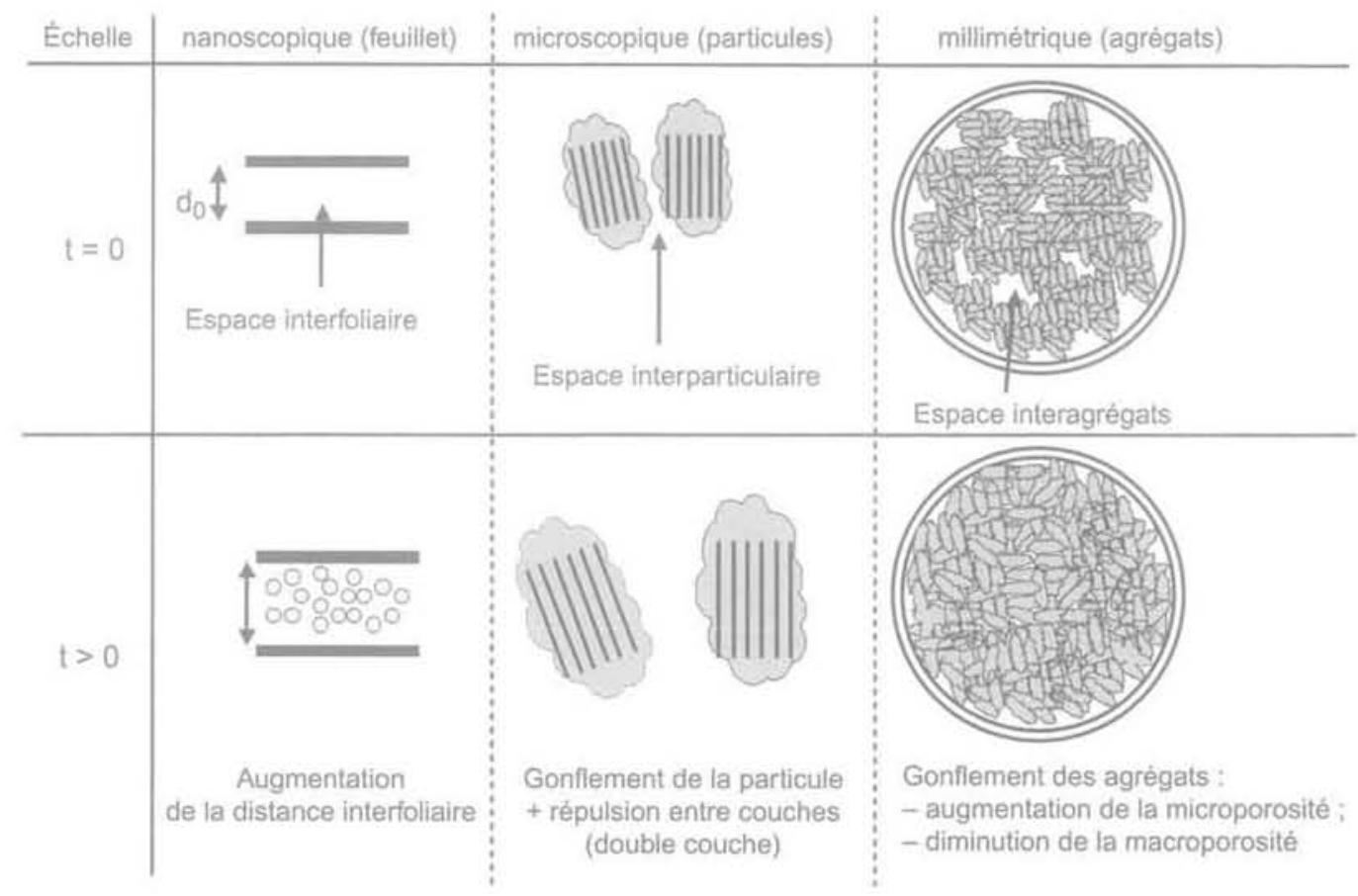

FiG. 16 Les différentes échelles du gonflement.

The different scales of the swelling.

Le cas de l'essai à volume constant reste un peu marginal: certes, la gamme de teneur en eau explorée demeure bien plus faible que pour les deux autres essais $(25 \% ; 30 \%)$, mais les valeurs prises par la diffusivité sont trois fois plus importantes, même à l'instant initial. Pour cet essai, on notera au passage que la notion de saturation a le mẻme sens que pour un matériau indéformable et que la quantité d'eau maximale pouvant pénétrer au sein du matériau est prévisible, conduisant à une teneur en eau à saturation, woil, voisine de $30 \%$.

Delage et al. (1998) ont travaillé sur des éprouvettes de bentonite fortement compactée. Selon eux, les changements internes de la microstructure durant l'hydratation à gonflement empèché, conduisent à des effets de colmatage des pores, qui ralentissent la progression du front d'humidification. D'autres auteurs avaient déjà formulé, voire vérifié cette hypothèse (Yong et Mohamed, 1992; Komine et Ogata, 1996). Dans notre cas, cela permet d'expliquer la faible variation de la teneur en eau au sein de l'éprouvette, mais ne justifie pas que le front d'humidification ait une progression plus rapide.

Sur la figure 17, relative à l'essai à volume constant, et représentant l'évolution temporelle de la pression de gonflement exercée par l'éprouvette dans le sens axial, on voit une augmentation très brusque au tout début de l'essai d'imbibition. Cela permet d'imaginer que les contraintes mécaniques imposées par le matériau sur lui-même interviennent de manière importante dans le transfert de l'eau. Rien n'interdit de penser que la diffusivité suive une croissance analogue à cette courbe de pression au voisinage de l'instant initial. Le modèle exponentiel n'est alors évidemment plus adapté pour décrire un éventuel saut de diffusivité aux tout premiers instants de l'imbibition. Une meilleure connaissance microscopique du matériau et le choix d'un modèle de diffusivitè plus complexe permettront sans doute de mieux appréhender ce type de mécanisme. Quoi qu'il en soit, ce dernier résultat, assez surprenant, mériterait d'être confirmé par une nouvelle expérience menée dans des conditions parfaitement similaires.

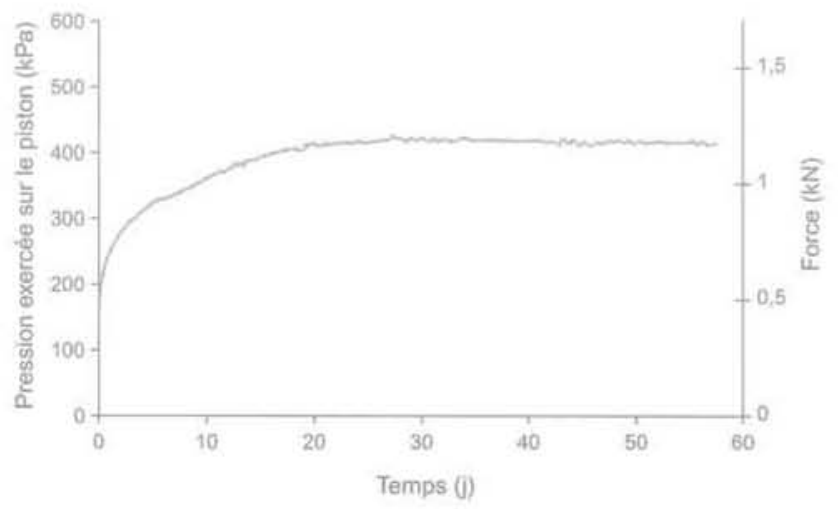

FIG. 17 Évolution de la pression de gonflement dans le sens axial en fonction du temps (essai à volume constant).

Swelling pressure in the axial direction versus time (constant volume test). 


\section{Conclusion}

Ce travail aborde la caractérisation hydraulique des sols argileux gonflants en relation avec les effets de couplage hydromécanique. Après avoir détaillé la composition de notre échantillon et la mise en forme des échantillons ainsi que les différents éléments du dispositif expérimental, des résultats d'ímbibition capillaire pour trois modes de confinement (libre, œdométrique et à volume constant) sont présentés et comparés. L'utilisation de la gammamétrie double-source et le recours aux techniques inverses s'avèrent pertinents dans l'identification de la diffusivité hydraulique des échantillons de sols considérés.

L'influence du confinement sur les cinétiques d'infiltration est clairement mise en évidence: plus le degré de confinement est important, plus la cinétique d'imbibition est lente. En terme de diffusivité matérielle, le cas des essais libre et œdométrique peut s'expliquer de manière cohérente. En revanche, l'interprétation de l'essaí d'imbibition à volume constant est encore difficile: certaines courbes comme l'évolution temporelle de la pression de gonflement axiale semblent indiquer que les contraintes mécaniques, très fortes en début d'essai conditionnent de manière très importante le transfert hydrique.

Le bilan de ce travail montre que l'utilisation de l'équation de Philip (1968) et de Smiles et Rosenthal (1968) que nous faisons est un peu trop simpliste pour décrire les transferts hydriques dans un sol gonflant non saturé soumis à des contraintes mécaniques. Le concept de diffusivité ne doit cependant pas être rejeté car les ordres de grandeur sont malgré tout conservés.

Pour aller plus loin dans l'analyse des résultats expérimentaux, il faudrait probablement raffiner la description physique du problème en envisageant par exemple une expression de la diffusivité matérielle qui dépende à la fois de la teneur en eau mais également du tenseur des contraintes.

\section{REMERCIEMENTS}

Nous tenons à remercier blen cordialement Monsieur A. Mesbah de l'ENTPE à Vaulx-en-Velin, pour l'aide apportée dans la mise en ceuvre des éprouvettes de sol (compactage double-piston), ainsi que Monsieur J. Yvon du I EM à Vandoeuvre-lés-Nancy, pour l'anaJyse minéralogique de la bentonite.

\section{Bibliographie}

Angulo R. - Caractérisation hydrodynamique de sols déformables partiellement saturés: étude expérimentale á l'aide de la spectroscopie gamma double-source. Thèse Mécanique, INPG Grenoble, 1989.

Angulo R. Gauder J.P., Thony J.L., Vauclin M. - "Détermination experimentale des caractéristiques hydrodynamiques d'un sol gonflant non saturé pour la modélisation des écoulements m. Revue française de géotechnique, $n^{\circ} 62$. 1993. p. 4957.

Augier F. Coumans W.J. Hugget A. Kaasschieter E. Fi $-a$ On the risk of cracking in clay drying $x_{\text {. Chemical Enginee- }}$ ring Journal, vol. 86, $n^{\circ} 1-2,2002$, p. 133 138.

Barataud F. Moyne C., Stemmelen D. (cMeasurement of soil water diffusivity of an undisturbed forest soil using duajenergy gamma radiation technique 1. Soil science, vol. $164, n^{\circ}$ 7. 1999, p. 493 502.

Corey J.C., Peterson S.F., Wakat M.A. Measurement of attenuation of ${ }^{\mathrm{IT}} \mathrm{Cs}$ and 211Am gamma rays for soil density and water content determinations. Proceed. Soil Science Society of America, vol. 35, 1971, p. 215-219.

Delage P., Cui Y.J.. Yahia-Aissa M., Laure (de) E. - "On the unsaturated hydraulic conductivity of a dense compacted bentonite s. Proceed. of the Second International Conference on Unsaturated Soils/Unsat'98, Beijing, China, 2730 August 1998, Beijing, International Academic Publishers. vol. 1, 1998, p. 344-349.

Derriche Z., Iguechtal L., Tas M. - "Comportement des ouvrages dans les argiles expansives d'In-Aménas $n$, Revue fran- çaise de géotechnique, n 89, 1999. p. 55 65.

Gardner W.H. Campbell G.S.. Calissendorf C. - "Systematic and random errors in dual gamma energy soil bulk density and water content measurements 1), Soil Science Society of America, vol. 36, 1972, p. 393-398.

Garnier P., Angulo-Jaramilio R., Dicario D., Bauters T.W.J., Darnault C.J.G., Steenhuis T.S. Parlange J.Y.. Baveye P. a Dual-energy synchrotron $X$ ray measurements of rapid soil density and water content changes in swelling soils during infiltration $\mathrm{x}$. Water resources research, vol. $34, n^{*} 11,1998$, p. 2837 2842.

Kirby J.M. Smiles D.E. - a Comment on "Dual-energy synchrotron $X$ ray measurements of rapid soil density and water content changes in swelling soils during infiltration" by Patricia Garnier et al. x Water resources research, vol. $35, \mathrm{n}^{\circ} 11$, 1999, p. 3585-3587.

Komine P., Ogata N. - " Observation of swelling behaviour of bentonite by new electron microscope o. ICEG ISOsaka'96, International Congress on Environmental Geotechnics 2. Osaka, Japan, Nov. 1996. Rotterdam: Balkema, 1996, p. $563-568$.

Mesbah A., Morel J.C., Olivier M. - « Comportement des sols fins argileux pendant un essai de compactage statique: détermination des paramètres pertinents 1 . Materials and structures, London. 1992, vol, 32, 1999, p. 687-694.

Mitchell J.K. - Fundarnentals of soil behavior. New York, John Wiley \& Sons, Inc. 1993 (2nd ed.).

Nakano M. Amemiya Y., Fujii K. - « Satu- rated and unsaturated hydraulic conductivity of swelling clays $\%$. Soil science, vol. 141, $\pi^{\circ} 1,1986$, p. 1-6.

Philip J.R. $-\alpha$ Kinetics of sorption and volume change in clay-colloid pastes $\%$. Australian Journal of Soil Research, vol. 6. 1968, p. 249-267.

Philipponnat G. $-\alpha$ Retrait-gonflement des argiles, proposition de méthodologie 1. Revue française de géotechnique, $n^{\circ} 57$, 1991. p. 5-22.

Press W.H., Flannery B.P., Teukolsky S.A., Vetterling W.T. - Numerical Recipes: The Art of the Scientific Computation. New York: Cambridge University Press, 1986.

Rolland S. - Transfert hydrique dans des sols argileux qonflants : influence du confinement. Thése de mècanique énergétique, INPL Nancy, 2002.

Smiles D.E., Rosenthal M.J. - « The movement of water in swelling materials $\mathrm{H}$. Australian Journal of Soil Research, vol. 6. 1968, p. 237-248,

Tabani P. - Transfert hydrique dans des sols déformables. Thèse génie civil, hydrosytèmes, géotechnique, INPL Nancy, 1999,

Vandangeon $\mathrm{P}$, - $\alpha$ Exemples de sinistres en région parisienne ${ }^{2}$. Revue française de géotechnique, n 58, 1992, p. 7-14.

Villar M.V., Lloret A. - V Variation of the intrinsic permeability of expansive clays upon saturation $„$. Clay science for engineering Adachi \& Fukue (eds), Rotterdam, Balkema, 2001, p. 259-266.

Yong R.N. Mohamed A.M.O. - $\varangle$ A study of particle interaction energies in wetting of unsaturated expansive clays . $_{\text {. }}$ Canadian geotechnical journal, vol. 29 , $n^{\circ} 6,1992, p, 1060-1070$. 